\title{
The ratio of species of Atlantic and Pacific origin in modern Arctic fauna of bivalve molluscs
}

\author{
E.M. Krylova', D.L. Ivanov'², A.N. Mironov' \\ ${ }^{1}$ P.P. Shirshov Institute of Oceanology, Russian Academy of Sciences, Nakhimovskyi Prospect 36, \\ Moscow 117997, Russia; e-mail: elen@ocean.ru; miron@ocean.ru \\ 2 Zoological Museum, Moscow State University, Bolshaya Nikitskaya Str. 6, 125009 Moscow, \\ Russia; e-mail:dima_lumberg@mail.ru
}

ABSTRACT. The present work aims to estimate the ratio of species of the bivalve molluscs of the Atlantic and the Pacific origin in different geographical and bathymetrical regions of the Arctic basin. Seven shallow-water regions of the Arctic, with depths of 0-300 m, (the Norwegian, Barents, Kara, Laptev, East Siberian, Chukchi and Beaufort Seas) and three deep-sea regions with depths exceeding $1000 \mathrm{~m}$ (the Norwegian and Greenland Basins, Nansen and Amundsen Basins, Canada Basins) are considered. For the identification of origin we combined fossil records with records of the distribution of living genera and, when possible, used molecular genetic data. The distribution patterns of 265 species from 132 genera were analysed.

The share of species of Atlantic origin decreases in marginal Eurasian seas from the Norwegian Sea to the Chukchi Sea, and then increases in the Beaufort Sea. The share of species of Pacific origin is higher only in the Chukchi and Beaufort Seas, the species of the Atlantic and the Pacific origin are equally represented in the East-Siberian Sea. The share of species of the Atlantic origin is much higher in all deep-sea regions.

Many taxa of bivalves that dispersed into the Arctic from the North Pacific also penetrated into the North Atlantic. In contrast, most taxa of the Atlantic origin remained in the Arctic Ocean, having stopped at the Bering Strait. Differences between the Arctic boundaries, the Arctic - Atlantic (the Greenland-Iceland-Faeroe Ridge) and the Arctic - Pacific (the Bering Strait) as barriers for dispersal are discussed.

KEY WORDS: Arctic Ocean, biogeography, Bivalvia, origin of fauna.

\section{Соотношение видов атлантического и тихоокеанского происхождения в современной арктической фауне двустворчатых моллюсков}

\author{
Е.М. Крылова ${ }^{1}$, Д.Л. Иванов², А.Н. Миронов ${ }^{1}$ \\ ${ }^{1}$ Институт океанологии им. П.П. Ширшова РАН, Нахимовский проспект, 36, 117997, \\ Россия. \\ 2 Зоологический музей Московского государственного университета им. М.В. Ломоносова, \\ ул. Большая Никитская, 6, Москва 125009, Россия.
}

РЕЗЮМЕ. Цель работы заключалась в оценке доли видов двустворчатых моллюсков атлантического и тихоокеанского происхождения в разных географических районах Арктики и на разных батиметрических горизонтах. Для анализа были выбраны 7 
мелководных районов Арктики с глубинами от 0 до 300 м: моря Норвежское, Баренцево, Карское, Лаптевых, Восточно-Сибирское, Чукотское и Бофорта, и 3 глубоководных района с глубинами более 1000 м: Норвежская и Гренландская котловины, котловины Нансена и Амундсена, а также Канадская котловина. Всего проанализировано распространение 265 видов из 132 родов. Вывод о районе вселения в Арктику для каждого рода был сделан на основе палеоданных, данных о современном распространении, а также были использованы опубликованные результаты молекулярных исследований.

Доля видов атлантического происхождения уменьшается в краевых морях от Норвежского до Чукотского моря, а затем несколько повышается в море Бофорта. В мелководных районах доля видов тихоокеанского происхождения превышает долю видов атлантического происхождения только в Чукотском море и море Бофорта; в Восточно-Сибирском море виды атлантического и тихоокеанского происхождения представлены равными долями. В глубоководных котловинах доля видов атлантического происхождения значительно превышает долю видов тихоокеанского происхождения.

Большая часть родов двустворчатых моллюсков, проникших в Арктику из Северной Пацифики, распространилась также и в Северной Атлантике. Напротив, большая часть родов атлантического происхождения осела в Арктическом океане и не преодолела Берингов пролив, не приняв участия в трансарктическом обмене. Обсуждаются различия между барьерными свойствами границ Арктика-Атлантика (Гренландско-Исландско-Фарерский порог) и Арктика-Пацифика (Берингов пролив).

КЛЮЧЕВЫЕ СЛОВА: Арктика, биогеография, двустворчатые моллюски, происхождение фауны.

\section{Introduction}

The present Arctic fauna has been mainly shaped by repeated migrations of species from the Pacific and the Atlantic. The migrations were strongly influenced by glacial events and were most intense during periods of warming and the opening of the Bering Strait. As Dodson et al. (2007) pointed out, patterns of dispersal and colonization through the Bering Strait were complex, covering long periods of time and repeated Bering Strait openings. At least four openings are now documented. Fossil-calibrated dates indicate the openings during the earlymid Miocene $\sim 16-17$ million years ago $(\mathrm{Ma})$, at the end of the middle Miocene $\sim 11-12 \mathrm{Ma}$ (Sher, 1999), near the end of the Miocene $~ 5.3$ Ma (Gladenkov et al., 2002; Gladenkov, Gladenkov, 2004), and in the Pliocene between 4.1 and 3.1 Ma (Marincovich, Gladenkov, 1999). An intense trans-Arctic faunal exchange started only after the fourth opening of the Bering Strait, at approximately 3.1 Ma (Dodson et al., 2007; Vermeij, 1991). During subsequent glacial and interglacial periods the Arctic fauna retreated to the south and then repeatedly invaded northern regions.

There is an agreement among authors that the predominant direction of the trans-Arctic exchange of faunas was from the North Pacific towards the North Atlantic (Soot-Ryen, 1932; Ekman, 1953; Nesis, 1963; MacNeil, 1965; Durham, MacNeil, 1967; Kafanov, 1974, 1979, 1987; Gladenkov, 1978; Vermeij, 1991). At the same time, there is a serious discrepancy about the relative role of taxa of Pacific and Atlantic origin in the modern Arctic fauna. Among different groups of benthic invertebrates, echinoderms have been studied in most detail as to their ocean of origin. Considering the Arctic echinoderms as a whole, Anisimova (1986) and Smirnov (1994) incline to the opinion that the 
Table 1. Cruises used as a source of material in the present work. Таблица 1. Рейсы, материалы которых были использованы в работе.

\begin{tabular}{|l|c|c|c|c|}
\hline \multicolumn{1}{|c|}{ Research Vessel } & Cruise & Year & Area of study & $\begin{array}{c}\text { Number of } \\
\text { stations with } \\
\text { studied material }\end{array}$ \\
\hline Sevastopol & 5 & 1957 & Norwegian Sea, $105-1690 \mathrm{~m}$ & 12 \\
\hline Sevastopol & 8 & 1958 & Norwegian Sea, $185-1860 \mathrm{~m}$ & 5 \\
\hline Sevastopol & 10 & 1958 & Norwegian Sea, $140-1950 \mathrm{~m}$ & 3 \\
\hline Sevastopol & 15 & 1959 & Norwegian Sea, $1000-1940 \mathrm{~m}$ & 4 \\
\hline Vodnik, RT-61 & 26 & 1968 & Barents Sea, $85-250 \mathrm{~m}$ & 3 \\
\hline Persey-3 & 14 & 1975 & North-Western Atlantic, $70-280 \mathrm{~m}$ & 8 \\
\hline Tunets & 105 & 1978 & Barents Sea, $245-250 \mathrm{~m}$ & 2 \\
\hline Dmitrii Mendeleev & 49 & 1993 & Kara Sea, $63-236 \mathrm{~m}$ & 5 \\
\hline
\end{tabular}

modern Arctic fauna is mainly of the Atlantic origin. Based on the fauna of starfish, Djakonov (1945) and Mironov and Dilman (2010) arrived at the conclusion that the North Pacific was the principal donor for shallow-water regions. For the deep-sea Arctic starfish it was shown that the half of the present genera are of Atlantic origin and another half of Pacific origin (Dilman, 2009). For other taxa, a predominantly Pacific genesis was suggested for fish (Andriyashev, 1939) and gastropods (Galkin, 1955), and a predominantly Atlantic origin for isopods (Kussakin, 1979).

Different opinions have been expressed on the origin of Arctic bivalves. Nesis (1965) and Kafanov (1974) suggested that the great majority of genera on the Arctic shelf are of Pacific origin. According to Filatova (1957a), the modern fauna of the Arctic bivalves has been mainly formed by species of Atlantic origin and autochthonous species. Fedyakov and Naumov (1987) supposed that more than a half the Arctic species are of Atlantic origin. Sirenko (2001) also believed that the impact of the Atlantic on the faunistic composition of Arctic Eurasian seas was higher than that of the Pacific. Some authors agree that the deep-sea Arctic bivalve fauna has Atlantic roots (Filatova, 1957a; Fedyakov, Naumov, 1987), however Bouchet and Warén (1979, tabl.3) found evidence of close relations of abyssal Arctic bivalves with the Pacific.

Although the origin of the recent Arctic bivalve fauna has been widely discussed, the relative importance of the Pacific and Atlantic elements in different Arctic regions remains mainly unknown. In the present paper we estimate the contribution of species of Pacific and Atlantic origin to the recent fauna of bivalves in different geographical and bathymetric regions of the Arctic. The origin of particular species was identified based on the biogeographical history of a genus. For the identification of origin, we use a complex approach combining fossil data with recent distribution of genera, and where possible the results of molecular genetics.

\section{Material and Methods}

\section{Definition of regions}

The boundaries of the Arctic Ocean are considered as passing along the northern limit ofDavis Strait and the Greenland-Iceland-Faeroe Ridge, with the Atlantic and the Bering Strait, separating the Arctic and Pacific (Leontjev, 1985). These geomorphological limits of the Arctic do not coincide with biogeographic boundaries (Briggs, 1995, figs. 82-86). Our biogeographic analysis was based on seven selected shallow-water regions of the Arctic, with depths of 0-300 m, and three deep-sea regions with depths exceeding $1000 \mathrm{~m}$. The shallow water regions include the Norwegian, Barents, Kara, Laptev, East Siberian, Chukchi and Beaufort Seas. The deep-sea regions include the western basins (Norwegian and Greenland Basins), the central basins (Nansen and Amundsen Basins) and the Canada Basin. Latitude $40^{\circ} \mathrm{N}$ was taken as the southern border of the North Atlantic and the North Pacific for the purposes of this study. 


\section{Species considered}

In this paper we use the term "Arctic species" for all species occurring in the Arctic Ocean without regard to a species range type. The list of Recent Arctic bivalve molluscs and data on their distribution was based mainly on literature sources: Gorbunov, 1946; Filatova, 1957a, b; Gladenkov, 1978; Bernard, 1979; Bouchet, Warén, 1979; Kafanov, 1979; Knudsen, 1985; Lubinsky, 1980; Fedyakov, Naumov, 1989; Warén, 1989, 1991; Brattegard, Holthe, 1997; Hansson, 1998; Gulliksen et al., 1999; Coan et al., 2000; Richling, 2000; Galkin, Voronkov, 2001; Sirenko, 2001; Kantor, Sysoev, 2005; Schiøtte, 2005; Sneli et al., 2005; Naumov, 2006; Sirenko et al., 2009; and others. Despite considerable gaps in the taxonomy and diversity of the Arctic bivalves, a significant step forward was made recently owing to the database efforts of the Zoological Institute of the Russian Academy of Sciences (www.zin.ru/ projects/arccoml/eng/dbases.htm). For information about taxonomic composition and distribution of bivalves from the northern Atlantic and the Pacific, we used the WoRMS Database (Appeltans et al., 2012) and the Database of Western Atlantic Marine Mollusca, together with literature sources (Coan et al., 2000; Scarlato, 1981; Scarlato, Kafanov, 1988). We also used original data obtained by the authors, based on material collected on the cruises listed in Table 1. The genera used in the Bivalvia are not consistent between different sources. Mainly we followed WoRMS but in some cases accepted other versions closest to our own opinions.

The final list of the bivalve taxa from the studied regions contains 265 species from 132 genera.

The total number of species common with the North Atlantic, the North Pacific, and the number of Arctic endemics were calculated for each of the seven shallow-water and three deepsea Arctic regions (Table 2; Figs 1, 2).

Data on the paleo-distributions was retrieved from the Paleobiology Database (http:// paleodb.org/), as well as from numerous literature sources (Gladenkov, 1978; Kafanov,
Ogasawara, 2003, 2004; Merklin et al., 1962, 1979; Svitoch, 2003 and others) and also from the database on bivalve molluscs of the European Neogene of R. Janssen (Senckenberg Forschungsinstitut und Naturmuseum, Frankfurt a. M.). We used data on fossil records, mainly from the northern regions of Europe, Asia and the North America, since the middle Miocene (Table 3). Overall 111 Recent genera (83\% of the modern Arctic fauna) are known from fossil findings in the northern regions.

\section{The source of bivalves penetrating into the Arctic Ocean}

By characterizing genera as of North Pacific or North Atlantic origin, we mean not the region of origin of the genus but the region from which the genus first penetrated the Arctic Ocean.

The source region (Atlantic or Pacific) was suggested by the biogeographical history of the genus. If a genus migrated from the North Pacific to the North Atlantic through the Arctic and then a species of this genus colonised the Arctic from the North Atlantic, it was accepted that this species (and the genus) is of the North Pacific origin. If an endemic Arctic species belonged to a genus of Atlantic or Pacific origin, then this species was accepted as being from the Atlantic or Pacific correspondingly. "Dual" origin of a genus, i.e. migration of the genus to the Arctic independently from the Pacific and the Atlantic, was adopted only if supported by paleo-data at the species level.

The conclusions about origin were made for each individual genus based on paleontological and neontological evidences. Both paleontological and neontological data provide only indirect evidence the colonisation pathway, only suggesting that one of the two pathways is more probable. Therefore there is a high probability of incorrect conclusions about the colonisation pathway of some genera. Nevertheless, analysis of numerous taxa increases the chance of revealing real trends in the biogeographical history of the Arctic.

In paleo-analysis, data on the Recent Arctic species, or species closely related to them, were 


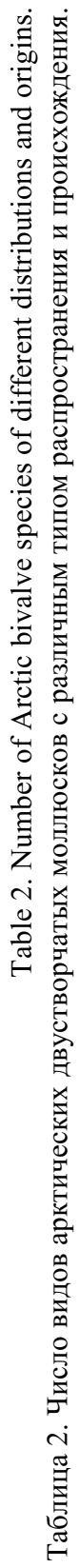

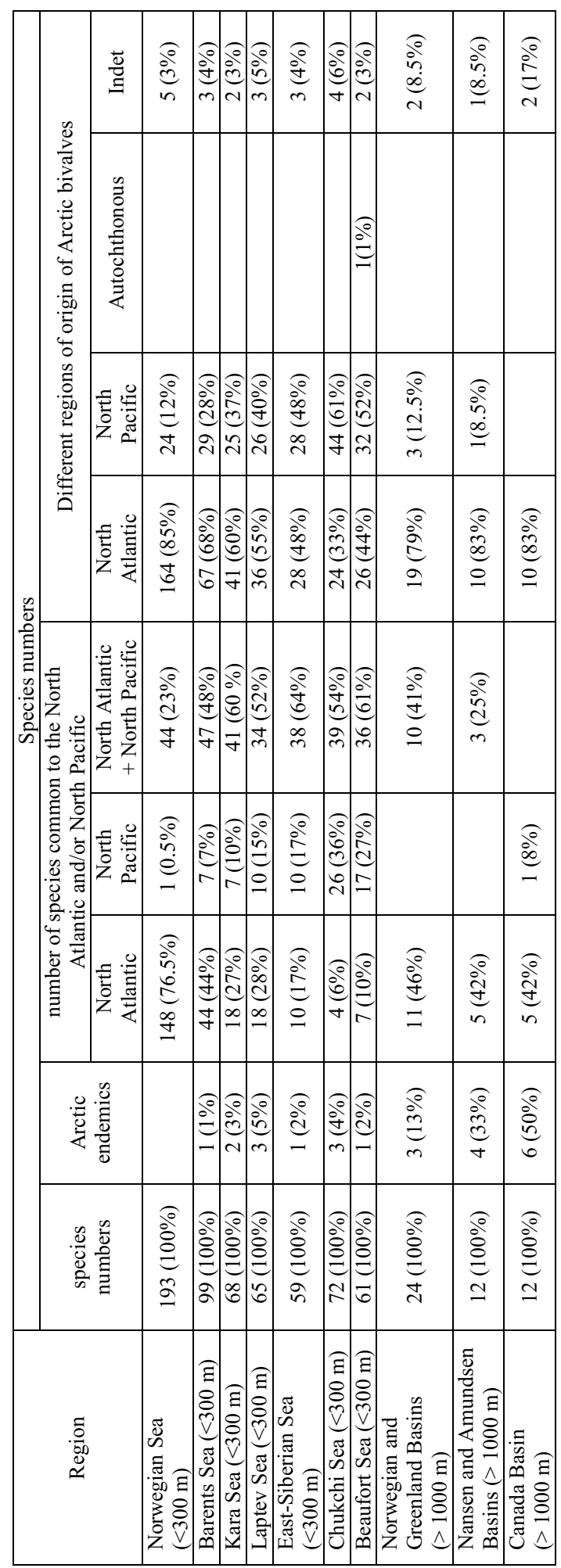




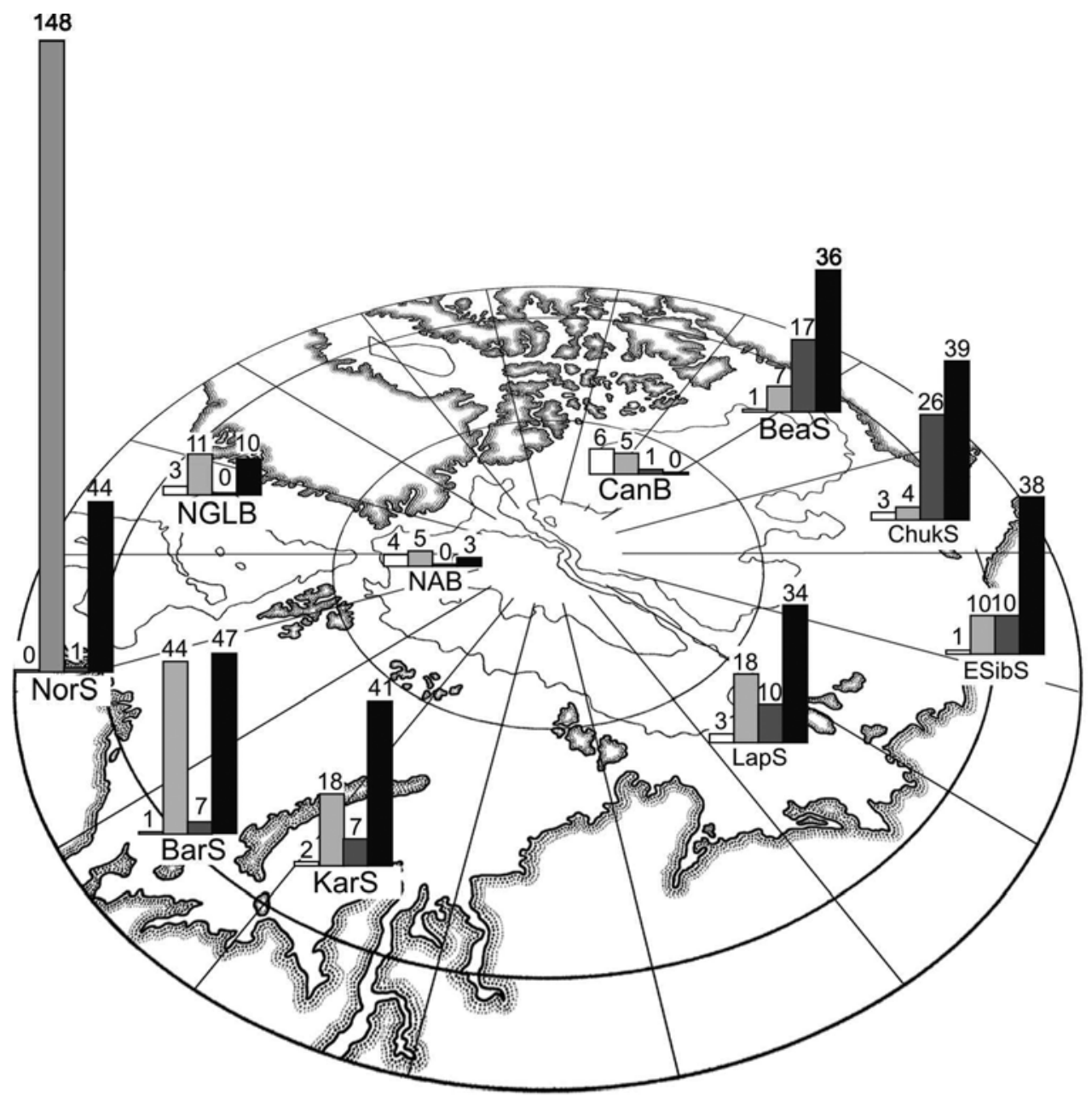

\section{Arctic endemics $\square$ Atlantic $\square$ North Pacific $\square$ Atlantic + North Pacific}

Fig. 1. Number of species of Bivalvia common with the North Atlantic and/or the North Pacific in different Arctic regions.

Abbreviations: NorS — Norwegian Sea; BarS — Barents Sea; KarS - Kara Sea; LapS — Laptev Sea; ESibS — East Siberian Sea; ChukS - Chukchi Sea; BeaS - Beaufort Sea; NGLB - Norwegian and Greenland Basins; NAB Nansen and Amundsen Basins; CanB - Canada Basin.

Рис. 1. Число видов двустворчатых моллюсков общих с Северной Атлантикой и (или) северной частью Тихого океана в различных регионах Арктики.

Обозначения: NorS - Норвежское море; BarS - Баренцово море; KarS — Карское море; LapS — море Лаптевых; ESibS - Восточно-Сибирское море; ChukS - Чукотское море; BeaS - море Бофорта; NGLB — Норвежская и Гренландская котловины; NAB - котловины Нансена и Амундсена; CanB - Канадская котловина.

taken into account first. If data on a Recent Arctic species was absent, information on all species in the genus was examined. Paleo-analysis was performed by two approaches, differing by the length of the period covering the paleo-data. In the first, the period from the Middle Miocene to the present time was considered. In the second, the period considered was from the Pliocene to the present. The first version could overestimate the number of genera of 


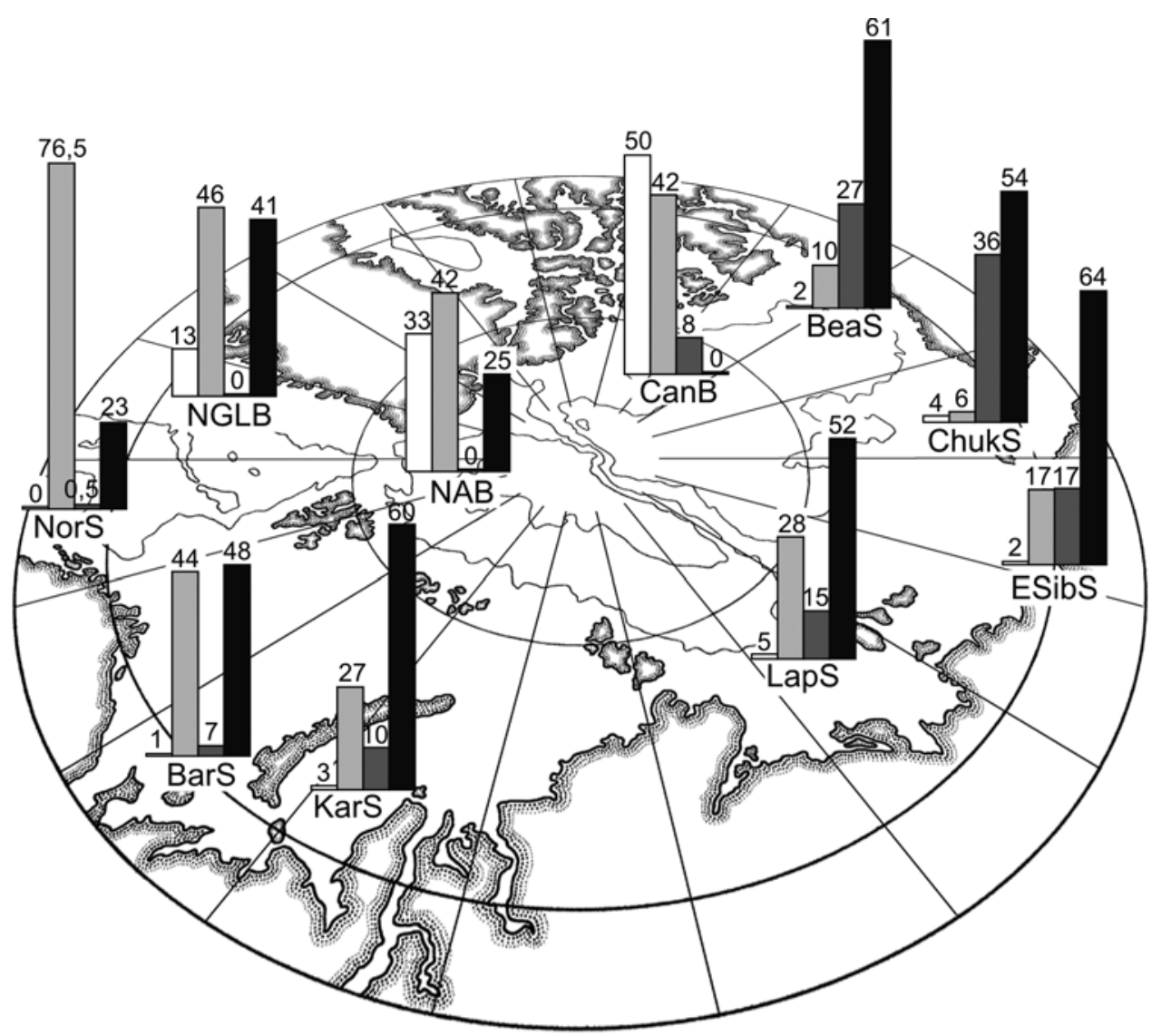

Arctic endemics $\square$ Atlantic $\square$ North Pacific $\square$ Atlantic + North Pacific

Fig. 2. Percentage of species of Bivalvia common with the North Atlantic and/or the North Pacific in different Arctic regions.

Abbreviations: see Fig. 1.

Рис. 2. Процентное соотношение видов двустворчатых моллюсков общих с Северной Атлантикой и (или) северной частью Тихого океана в различных регионах Арктики.

Обозначения те же, что на рис. 1.

Atlantic origin, because the Mediterranean Centre of Species Diversity (Mironov, 2006) still existed in the Miocene and fauna exchange occurred mainly through the low-latitude areas, from the Atlantic to the Pacific (Mironov, 2006). The second approach probably reflects the situation more adequately, because active transArctic exchange between faunas of the North Atlantic and the North Pacific began in the Middle Pliocene, i.e. about 3.1 Ma (Vermeij, 1991; Dodson et al., 2007). We assumed that a genus entered the Arctic from the area of its earliest record during the corresponding chosen period. If the record ages of a genus in the North Atlantic and the North Pacific were the same, and modern Arctic species of this genus are unknown in fossils, the region from where the genus penetrated the Arctic was considered as uncertain. There is principle possibility of a "dual colonization" scenario, i.e. when different species of the same genus have independently invaded the Arctic from the North Pacific and the North Atlantic without the trans-Arctic exchange. We accepted this scenario only for the 


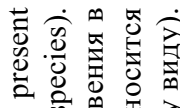
की

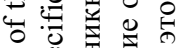

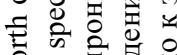

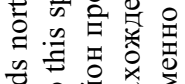

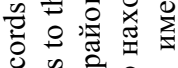
递 娄

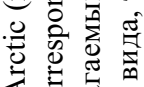

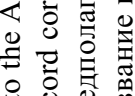
을 웝 월

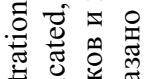

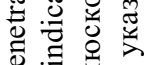

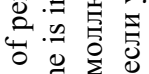

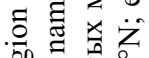
敋的数 可

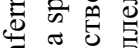
的兽尊

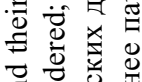

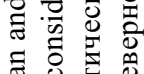
등 을 ․ํㄹㄹ 年亭 을

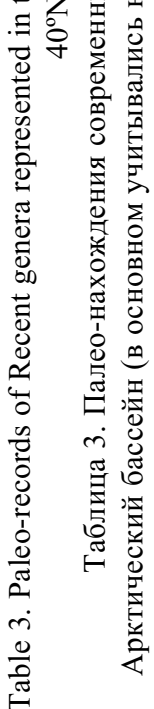

\begin{tabular}{|c|c|c|c|c|c|c|c|c|}
\hline 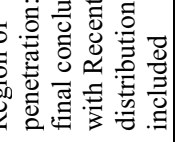 & 总 & 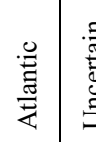 & 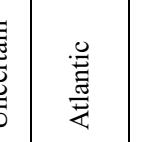 & 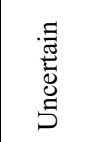 & 总 & 总 & 总 & 总 \\
\hline 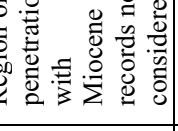 & 兹 & 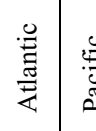 & 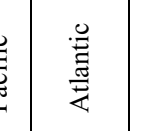 & 咅 & 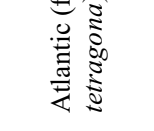 & 旁 & 总 & 产 \\
\hline$\frac{5}{5}=0$ & 总 & 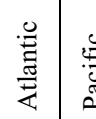 & 总 & 喽 & 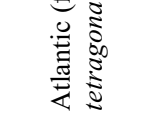 & 童 & 总总 & 产 \\
\hline 竞 & 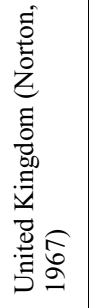 & & 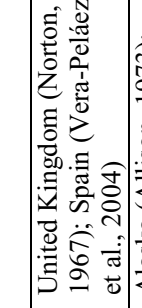 & & 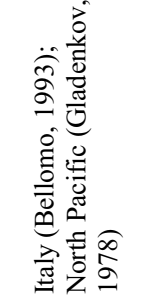 & 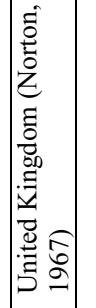 & 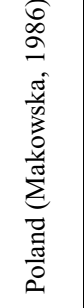 & 童 \\
\hline 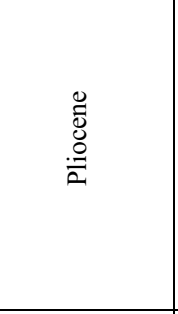 & 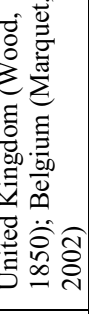 & & 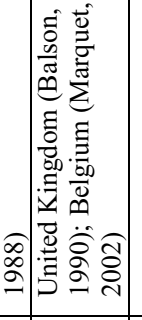 & 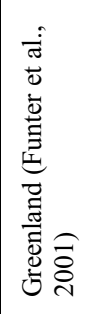 & 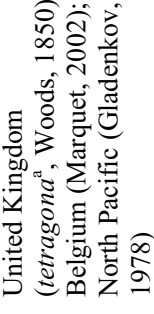 & & 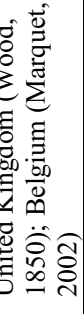 & \\
\hline 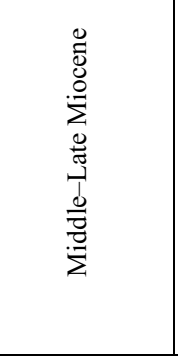 & 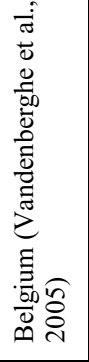 & 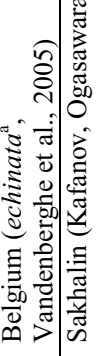 & 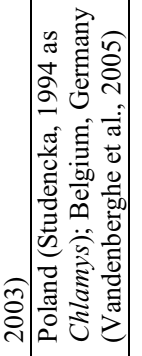 & & 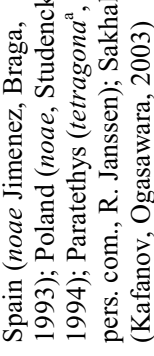 & & 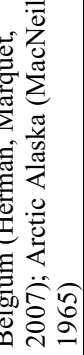 & 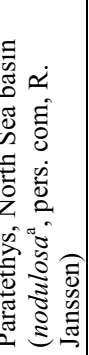 \\
\hline 善 & 蛋 & 言 & 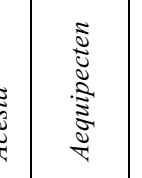 & עี & 苋 & है & हूँ & ถู้ \\
\hline & - & $\begin{array}{lll}- & -\end{array}$ & $1+$ & $n$ & 0 & -1 & $\infty$ & $\sigma$ \\
\hline
\end{tabular}


The ratio of species of Atlantic and Pacific origin of bivalve molluscs

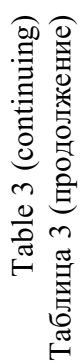

\begin{tabular}{|c|c|c|c|c|c|c|c|c|c|c|}
\hline 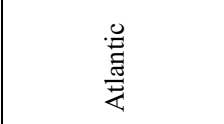 & 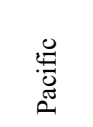 & 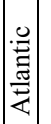 & 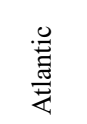 & 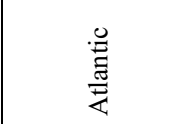 & 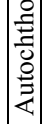 & 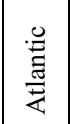 & 总 & 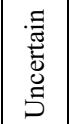 & 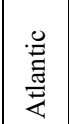 & 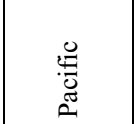 \\
\hline 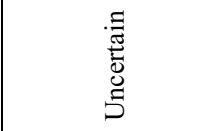 & $\begin{array}{l}\text { 总 } \\
\text { 焉 }\end{array}$ & $\mid \begin{array}{l}\frac{\pi}{\pi} \\
\frac{\pi}{\partial} \\
0 \\
z\end{array}$ & 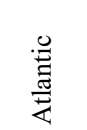 & $\frac{\text { 异 }}{\text { 壱 }}$ & 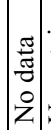 & 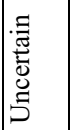 & 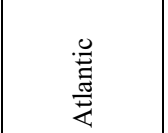 & 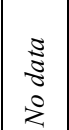 & 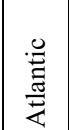 & $\begin{array}{l}\mathscr{U} \\
\mathscr{U} \\
\tilde{E}\end{array}$ \\
\hline 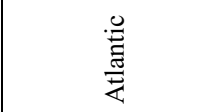 & $\begin{array}{l}\frac{\pi}{\pi} \\
\frac{\pi}{8} \\
0 \\
z\end{array}$ & 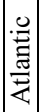 & $\begin{array}{l}\text { 岂 } \\
\text { 䲶 }\end{array}$ & 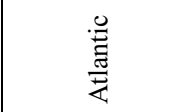 & 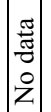 & 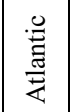 & $\begin{array}{l}\text { 䓌 } \\
\text { 惡 }\end{array}$ & 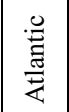 & 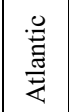 & 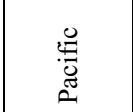 \\
\hline 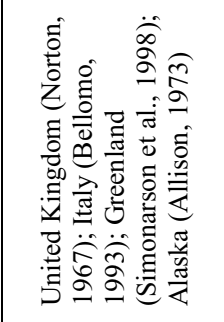 & 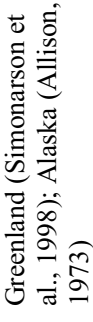 & & & 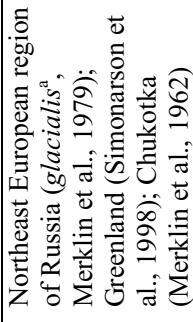 & & & 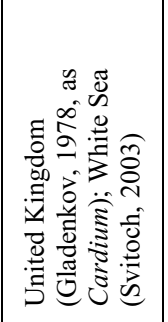 & & 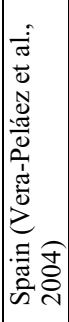 & 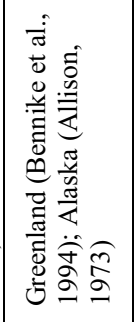 \\
\hline 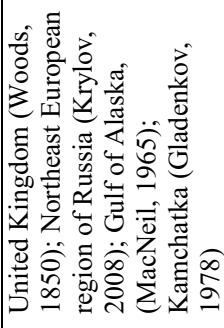 & 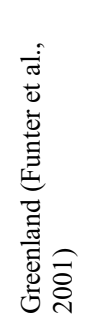 & & 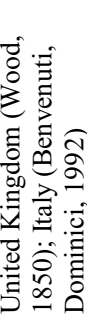 & 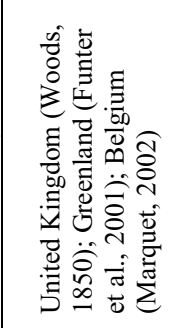 & & 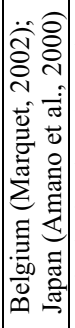 & 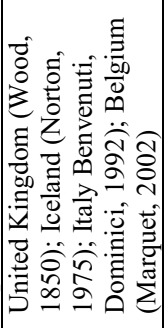 & & 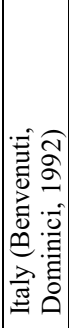 & 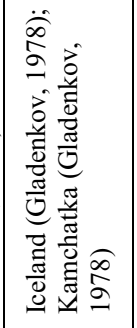 \\
\hline 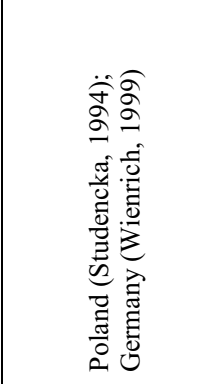 & & 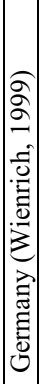 & 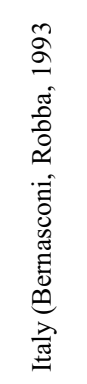 & 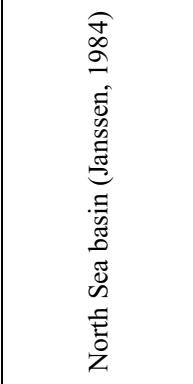 & & 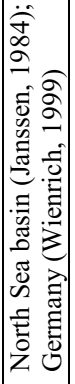 & 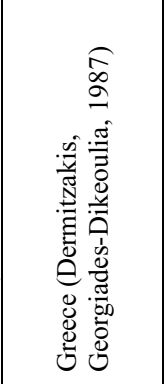 & 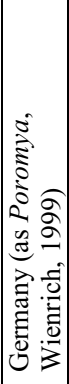 & 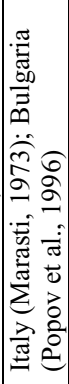 & 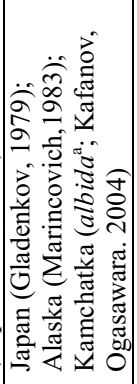 \\
\hline 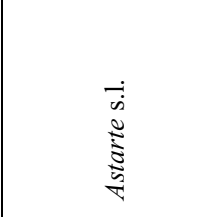 & 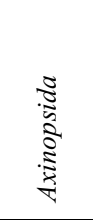 & $\mid$ & 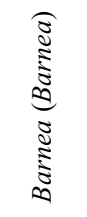 & 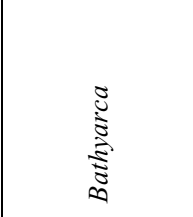 & $\mid$\begin{tabular}{c|}
0 \\
0 \\
0 \\
0 \\
$\vdots$ \\
$\vdots$ \\
0 \\
0
\end{tabular} & 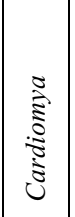 & 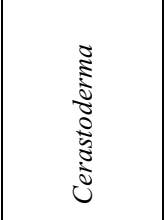 & ڤั) & 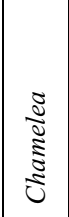 & 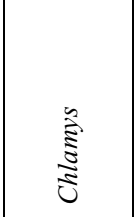 \\
\hline 으 & $=$ & $\cong$ & $\stackrel{m}{\sim}$ & \pm & $\because$ & $\stackrel{0}{0}$ & $I$ & $\stackrel{\infty}{-}$ & 2 & 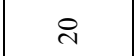 \\
\hline
\end{tabular}




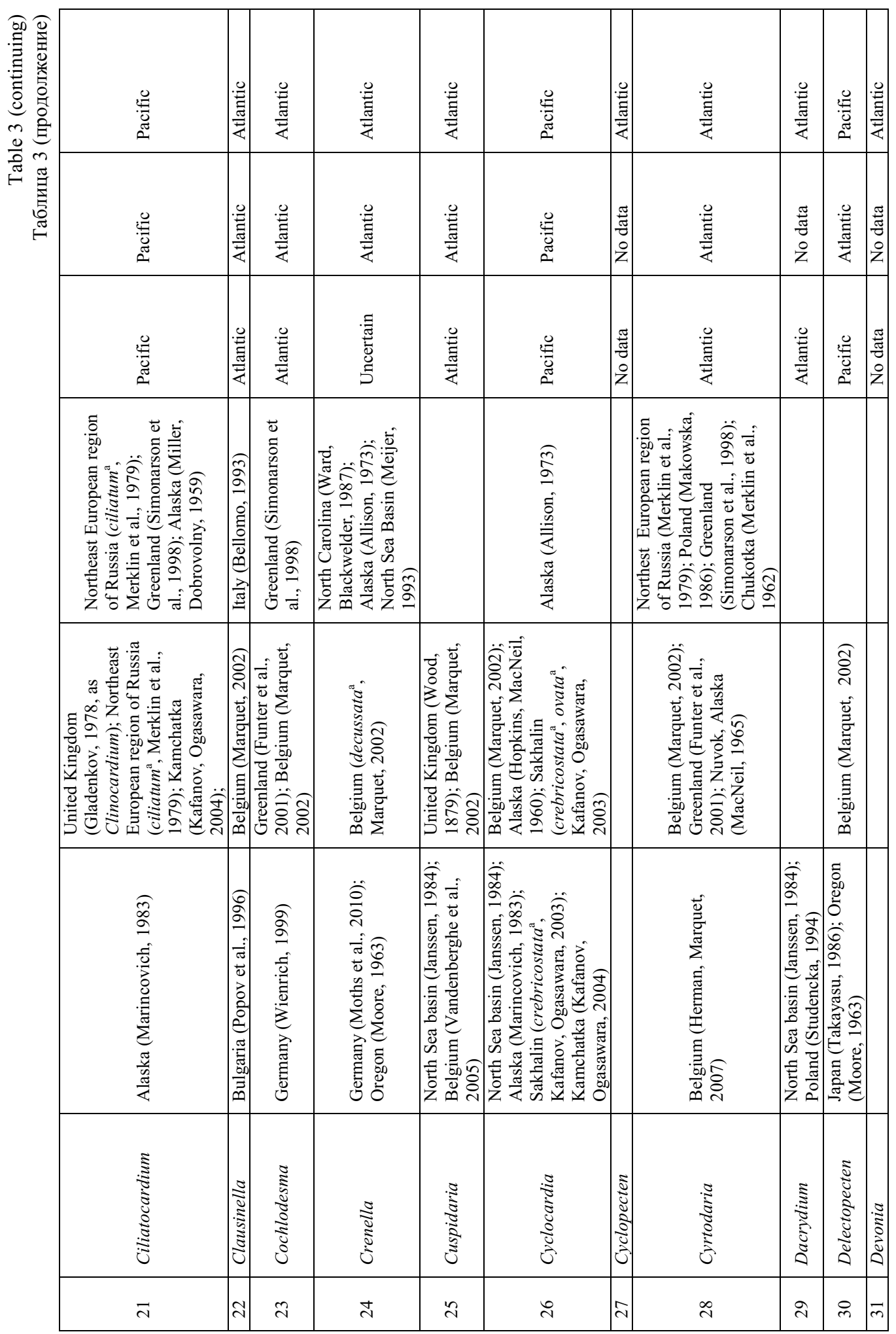




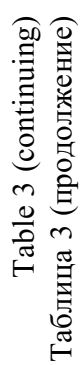

\begin{tabular}{|c|c|c|c|c|c|c|c|c|c|c|c|}
\hline 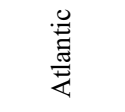 & 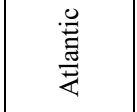 & 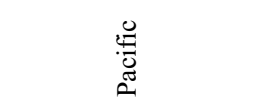 & 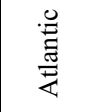 & 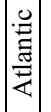 & 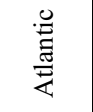 & 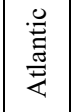 & 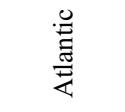 & 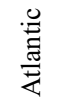 & 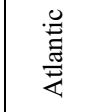 & 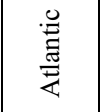 & 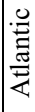 \\
\hline $\begin{array}{l}\text { 芑 } \\
\text { 志 } \\
\text { E }\end{array}$ & $\begin{array}{l}\text { 营 } \\
\text { 吾 }\end{array}$ & 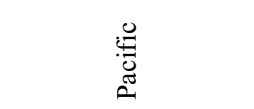 & 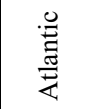 & $\mid$\begin{tabular}{|l|}
$\frac{\pi}{\tilde{g}}$ \\
0 \\
0 \\
$z$ \\
\end{tabular} & 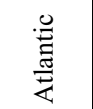 & 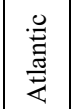 & $\begin{array}{l}\text { 导 } \\
\text { 壱 }\end{array}$ & $\begin{array}{l}\text { 节 } \\
\text { ¿ } \\
\text { z }\end{array}$ & 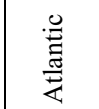 & $\begin{array}{l}\text { 总 } \\
\text { 莺 }\end{array}$ & 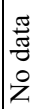 \\
\hline $\begin{array}{l}\text { 曾 } \\
\text { 焉 }\end{array}$ & 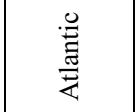 & 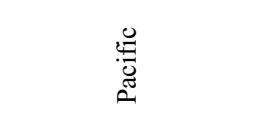 & 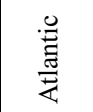 & $\mid \begin{array}{l}\frac{\pi}{\tilde{J}} \\
0 \\
0 \\
z\end{array}$ & 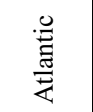 & 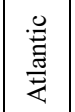 & $\begin{array}{l}\text { 䓌 } \\
\text { 䒿 }\end{array}$ & $\begin{array}{l}\text { 节 } \\
\text { ¿ } \\
\text { Z }\end{array}$ & 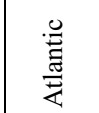 & 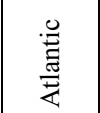 & 总 \\
\hline 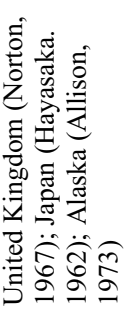 & & 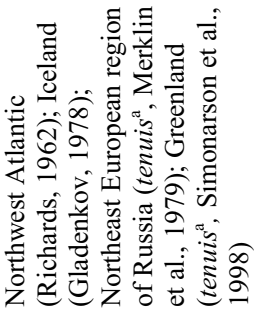 & 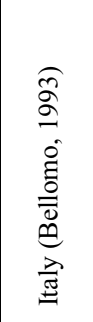 & & 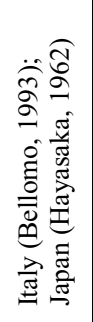 & & 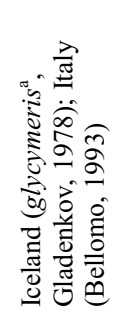 & 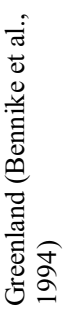 & 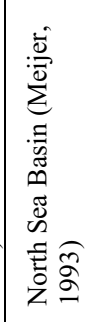 & 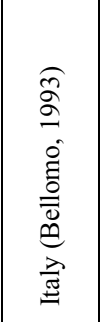 & \\
\hline 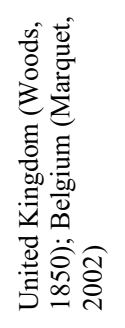 & 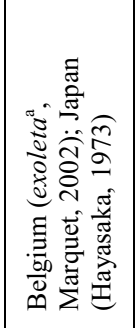 & 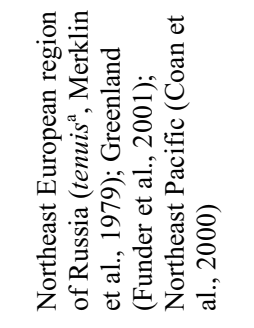 & 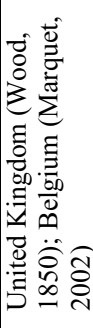 & & 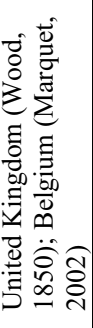 & 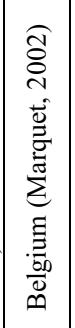 & 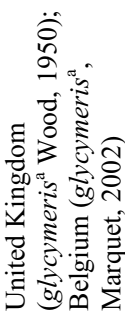 & & 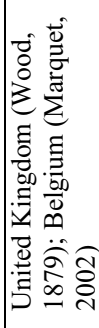 & 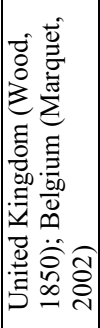 & \\
\hline 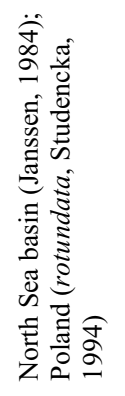 & 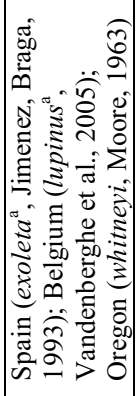 & 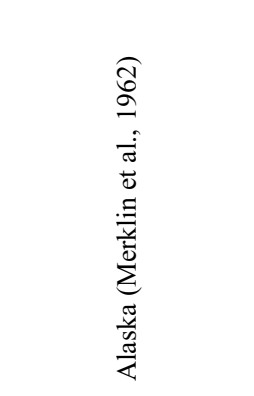 & 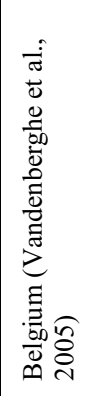 & & 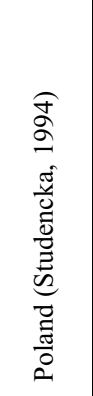 & 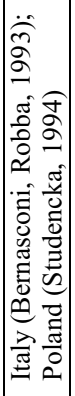 & 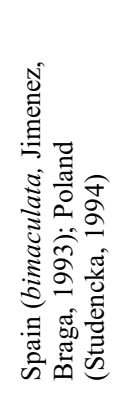 & & 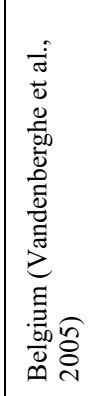 & 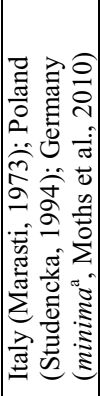 & 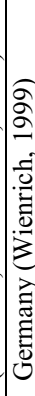 \\
\hline $\begin{array}{l}\frac{3}{0} \\
\frac{0}{0} \\
\frac{0}{0} \\
0\end{array}$ & 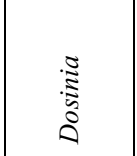 & 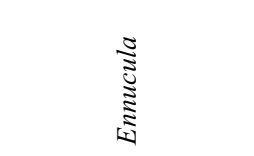 & 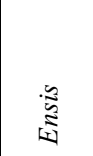 & 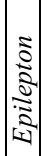 & క̃ & 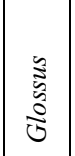 & 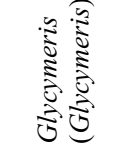 & 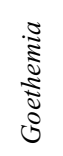 & 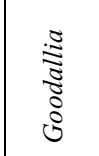 & $\begin{array}{l}\stackrel{\mathbb{Z}}{\mathbb{Z}} \\
\mathbb{0}\end{array}$ & \\
\hline లె & $m$ & ల్ & $m$ & 只 & $\hat{m}$ & $\stackrel{\infty}{m}$ & ले & q & $F$ & $\stackrel{\text { ㄱ }}{\text { s }}$ & \\
\hline
\end{tabular}




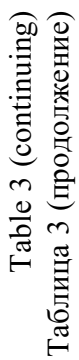

\begin{tabular}{|c|c|c|c|c|c|c|c|c|c|c|c|}
\hline 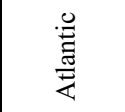 & $\begin{array}{l}\frac{\mathscr{E}}{E} \\
\frac{E}{E}\end{array}$ & 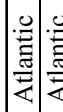 & 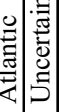 & $\begin{array}{l}\text { 号 } \\
\text { 莺 }\end{array}$ & 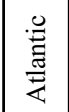 & $\begin{array}{l}\text { 异 } \\
\text { 恶 }\end{array}$ & $\begin{array}{l}\text { 莺 } \\
\text { 严 }\end{array}$ & 胥 & 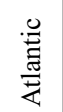 & 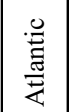 & 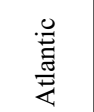 \\
\hline 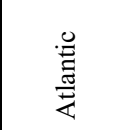 & 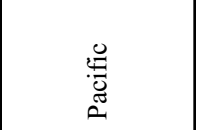 & 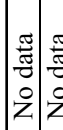 & 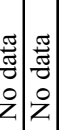 & $\begin{array}{l}\text { 䓌 } \\
\text { 莺 }\end{array}$ & 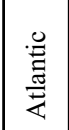 & $\begin{array}{l}\text { 总 } \\
\frac{\mathrm{E}}{\mathrm{E}}\end{array}$ & 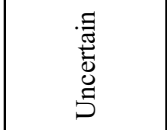 & $\begin{array}{l}\frac{\pi}{\pi} \\
\frac{\pi}{0} \\
0 \\
\text { z }\end{array}$ & 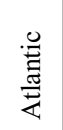 & $\begin{array}{l}\frac{\pi}{\tilde{J}} \\
\frac{\pi}{0} \\
\dot{z}\end{array}$ & 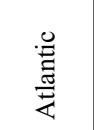 \\
\hline $\begin{array}{l}\text { 莺 } \\
\text { 焉 }\end{array}$ & $\begin{array}{l}\text { 莺 } \\
\frac{E}{E}\end{array}$ & & 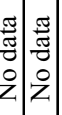 & $\begin{array}{l}\text { 莣 } \\
\text { 恶 }\end{array}$ & 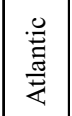 & $\begin{array}{l}\text { 芑 } \\
\frac{\mathrm{E}}{\mathrm{E}}\end{array}$ & $\begin{array}{l}\text { 总 } \\
\text { 恶 }\end{array}$ & 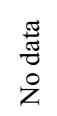 & 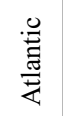 & $\begin{array}{l}\frac{U}{\vec{E}} \\
\frac{\vec{E}}{E}\end{array}$ & 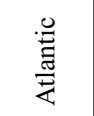 \\
\hline 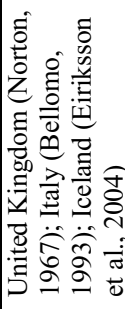 & 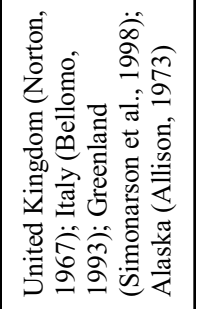 & & & 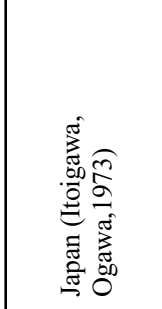 & 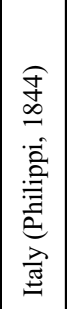 & 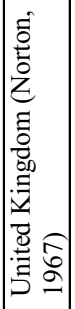 & 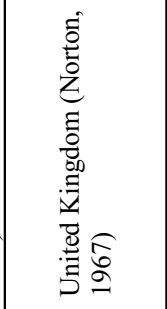 & & 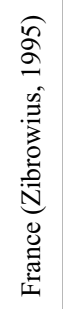 & & 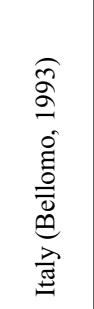 \\
\hline 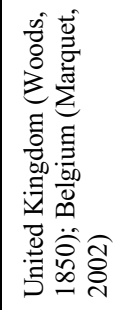 & 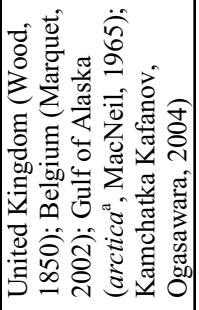 & & & 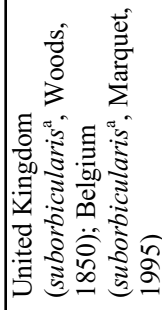 & 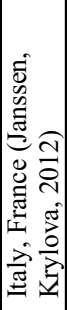 & 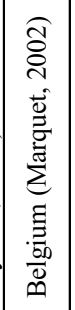 & 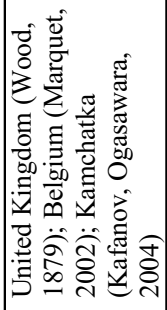 & & 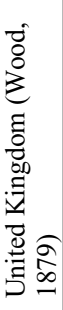 & & 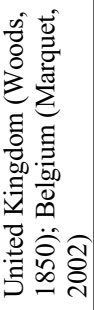 \\
\hline 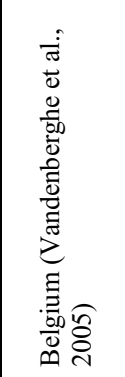 & 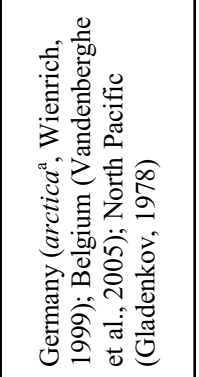 & & & 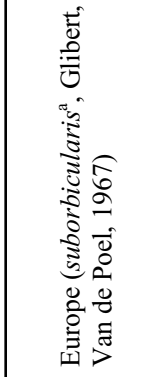 & 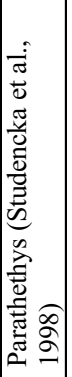 & 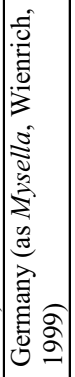 & 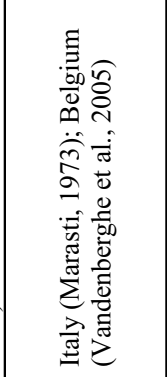 & & 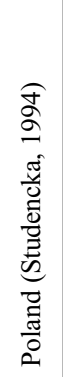 & 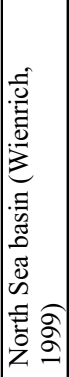 & 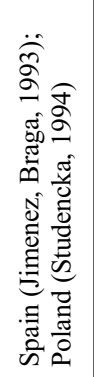 \\
\hline 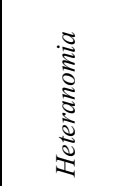 & 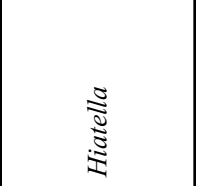 & 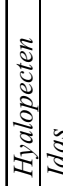 & 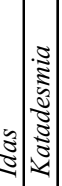 & 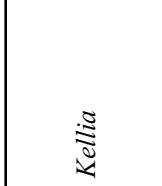 & 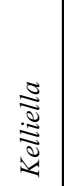 & & 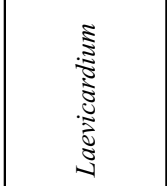 & इ્ّ & 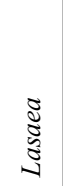 & 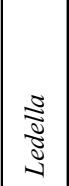 & 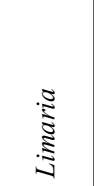 \\
\hline ナ & $\dddot{f}$ & 817 & $f \stackrel{\infty}{f}$ & g & 으 & $\bar{n}$ & ก & $n$ & $\dot{n}$ & $i n$ & $\stackrel{\circ}{n}$ \\
\hline
\end{tabular}


The ratio of species of Atlantic and Pacific origin of bivalve molluscs

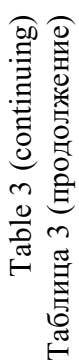

\begin{tabular}{|c|c|c|c|c|c|c|c|c|}
\hline $\begin{array}{l}\text { 莺 } \\
\text { 莺 }\end{array}$ & 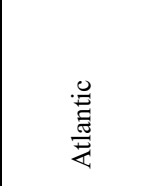 & 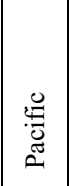 & $\begin{array}{l}\text { 岂 } \\
\text { 恶 }\end{array}$ & $\begin{array}{l}\text { 岂 } \\
\text { 䓌 } \\
\end{array}$ & 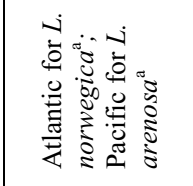 & 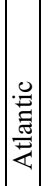 & 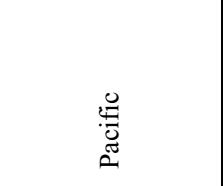 & 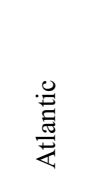 \\
\hline 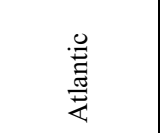 & 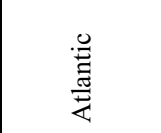 & 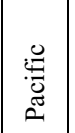 & 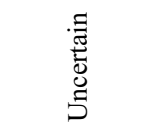 & 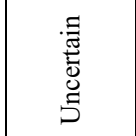 & 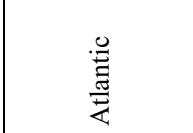 & \begin{tabular}{|l|} 
\\
$\frac{\pi}{\pi}$ \\
\\
2 \\
$z$
\end{tabular} & 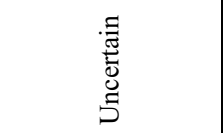 & $\begin{array}{l}\text { 总 } \\
\text { 䓌 }\end{array}$ \\
\hline 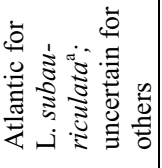 & 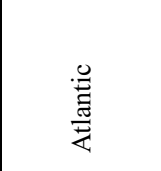 & 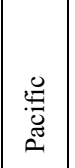 & 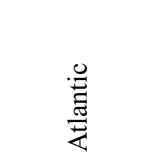 & 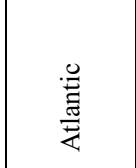 & 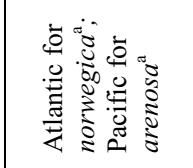 & 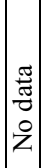 & 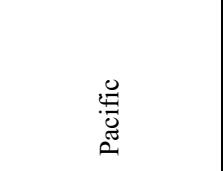 & 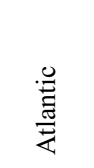 \\
\hline 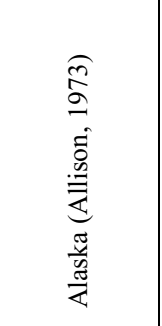 & 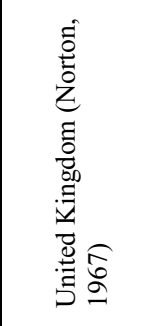 & 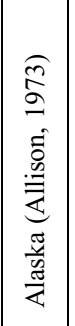 & 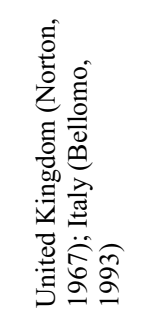 & 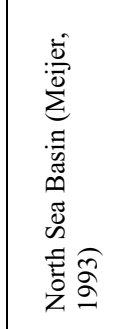 & 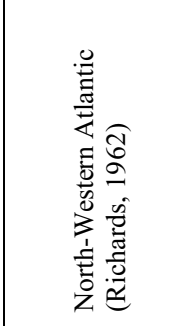 & & 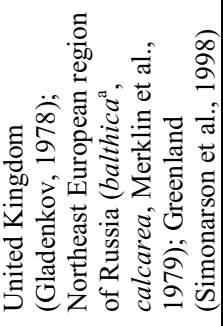 & 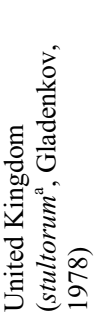 \\
\hline 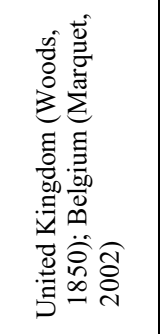 & 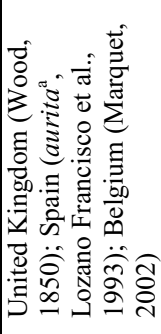 & 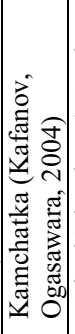 & 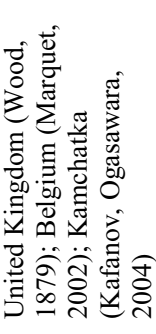 & 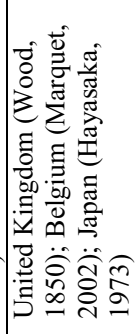 & 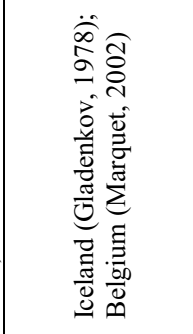 & & 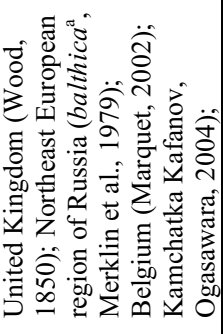 & 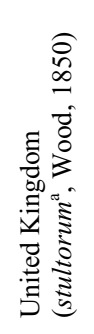 \\
\hline 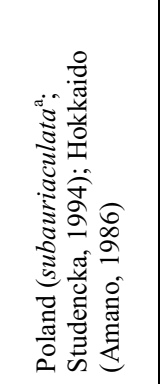 & 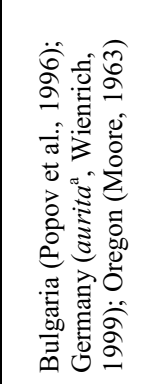 & 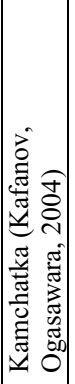 & 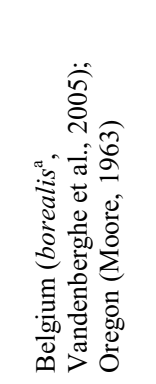 & 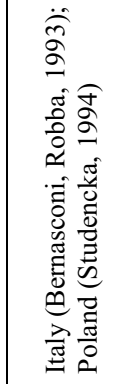 & 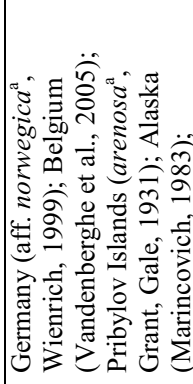 & & 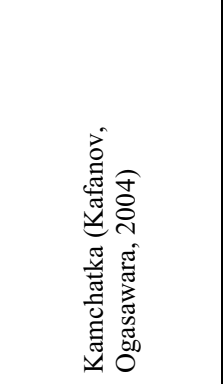 & 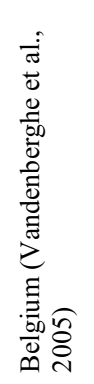 \\
\hline 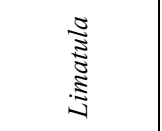 & 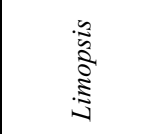 & 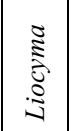 & 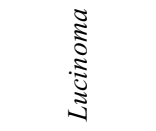 & 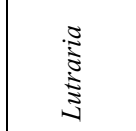 & $\begin{array}{l}\text { ڤ్ } \\
\vdots \\
\vdots \\
\vdots\end{array}$ & 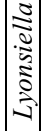 & 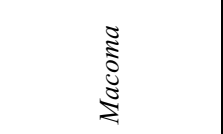 & 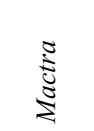 \\
\hline in & $\stackrel{\infty}{n}$ & in & 8 & $\bar{\sigma}$ & గె & $\hat{6}$ & $\vec{b}$ & 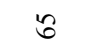 \\
\hline
\end{tabular}




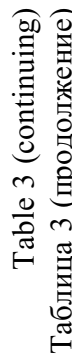

\begin{tabular}{|c|c|c|c|c|c|c|c|c|c|c|}
\hline$\underset{\mathscr{E}}{\mathscr{E}}$ & 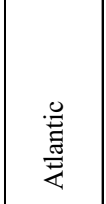 & $\begin{array}{l}\text { 总 } \\
\text { 焉 }\end{array}$ & 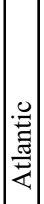 & $\begin{array}{l}\text { 莺 } \\
\text { 丵 }\end{array}$ & 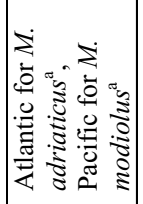 & 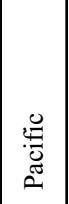 & $\begin{array}{l}\text { 曾 } \\
\text { 焉 }\end{array}$ & 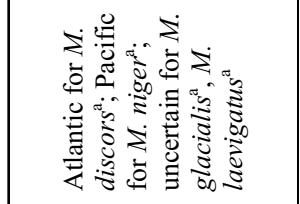 & $\begin{array}{l}\mathscr{D} \\
\stackrel{\tilde{D}}{0} \\
0\end{array}$ & \\
\hline 苞 & $\begin{array}{l}\text { 䔍 } \\
\text { 㐏 }\end{array}$ & $\begin{array}{l}\text { 总 } \\
\text { 焉 }\end{array}$ & 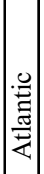 & $\begin{array}{l}\text { 莺 } \\
\text { 㐏 }\end{array}$ & 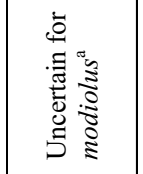 & 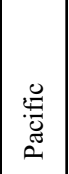 & 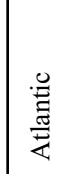 & 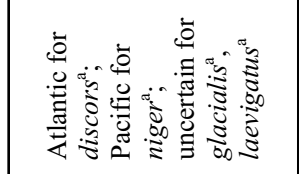 & 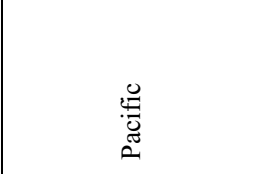 & $\begin{array}{l}\text { 苞 } \\
\text { Z } \\
\text { Z }\end{array}$ \\
\hline 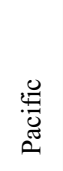 & 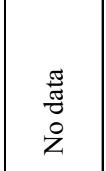 & 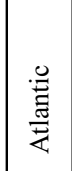 & 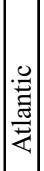 & $\begin{array}{l}\text { 䔍 } \\
\text { 㐏 }\end{array}$ & 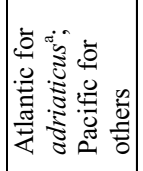 & 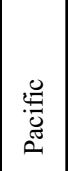 & $\begin{array}{l}\text { 芑 } \\
\text { 恶 }\end{array}$ & 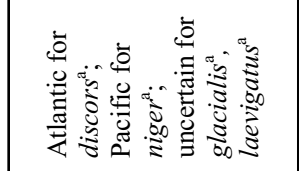 & 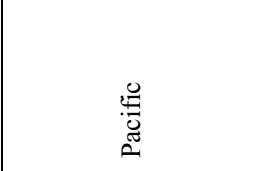 & \\
\hline 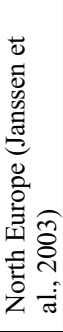 & & 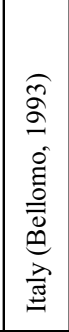 & & 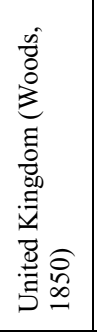 & 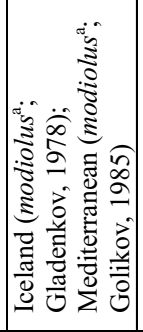 & 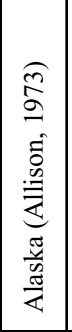 & 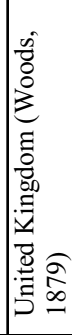 & 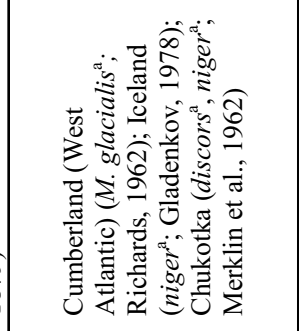 & 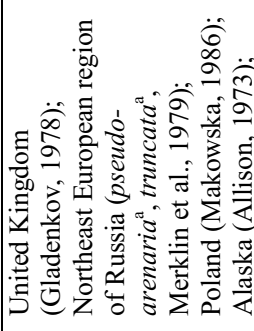 & \\
\hline 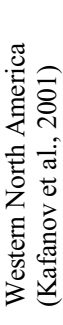 & 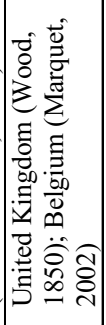 & 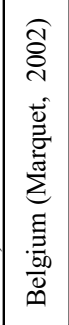 & 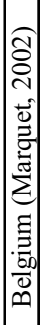 & 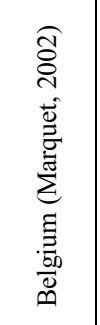 & 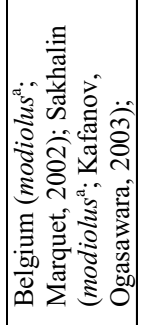 & 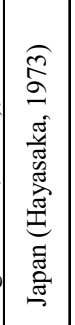 & 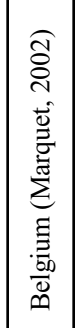 & 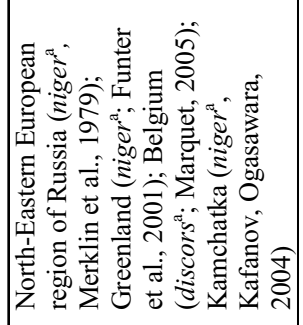 & 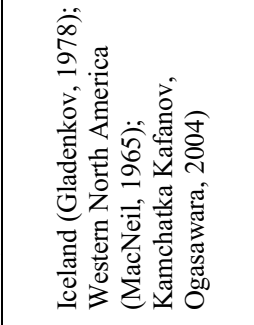 & \\
\hline 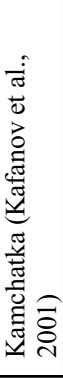 & & 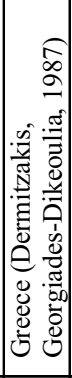 & 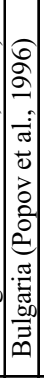 & 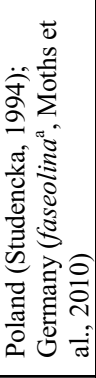 & 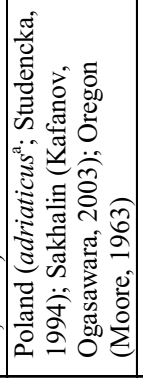 & 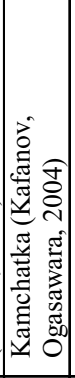 & 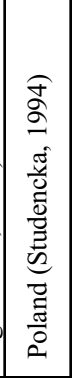 & 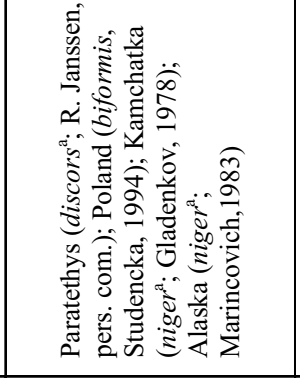 & 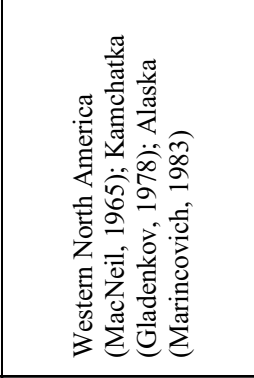 & \\
\hline 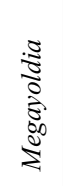 & 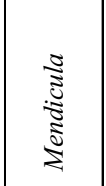 & 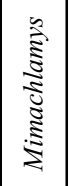 & 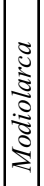 & 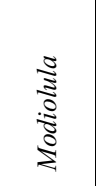 & 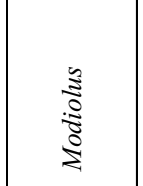 & 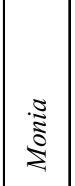 & 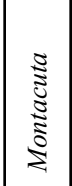 & 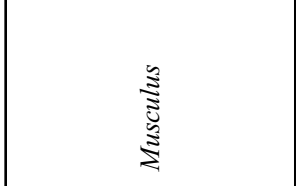 & $\stackrel{\Sigma}{\Sigma}$ & \\
\hline ๑ & $\hat{\sigma}$ & $\ddot{0}$ & 8 & 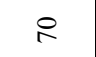 & $\nabla$ & $\approx$ & $\approx$ & 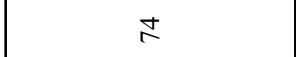 & 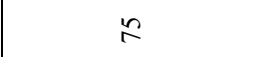 & \\
\hline
\end{tabular}




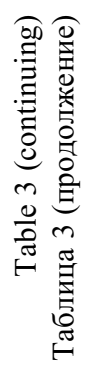

\begin{tabular}{|c|c|c|c|c|c|c|c|c|c|}
\hline $\begin{array}{l}\text { 总 } \\
\text { 焉 } \\
\end{array}$ & 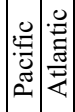 & 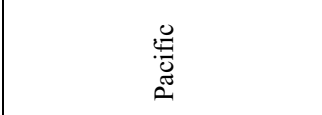 & 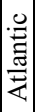 & 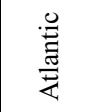 & 总 & 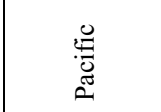 & & 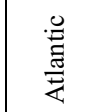 & 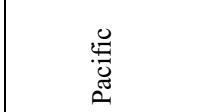 \\
\hline $\begin{array}{l}\frac{\pi}{\tilde{J}} \\
0 \\
z\end{array}$ & 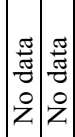 & 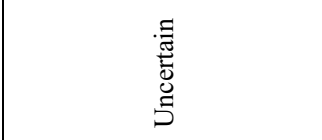 & \begin{tabular}{|l|} 
\\
$\frac{\pi}{\tilde{g}}$ \\
0 \\
$z$ \\
$z$
\end{tabular} & 兽 & 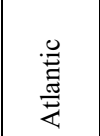 & 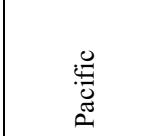 & 曾 & 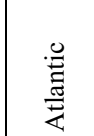 & 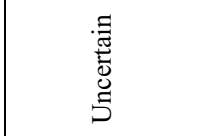 \\
\hline \multirow[t]{3}{*}{ } & & 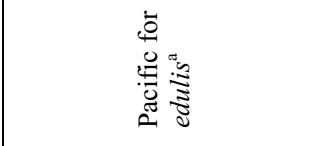 & $\mid$\begin{tabular}{|l}
$\frac{\pi}{\tilde{J}}$ \\
0 \\
0 \\
$Z$ \\
\end{tabular} & 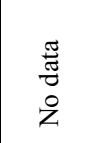 & 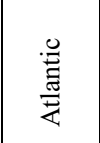 & 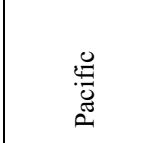 & $\begin{array}{l}\text { 足 } \\
\text { 严 }\end{array}$ & 总 & 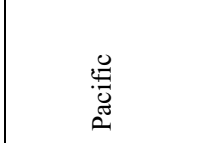 \\
\hline & & 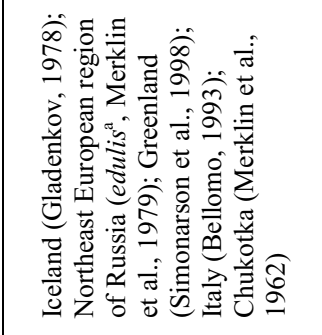 & & 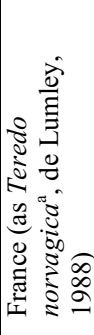 & 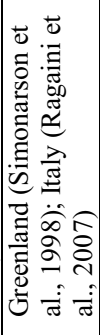 & 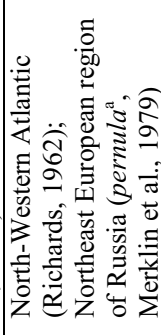 & 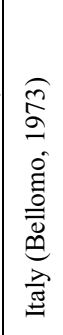 & 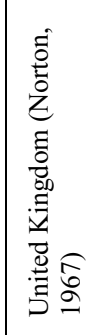 & 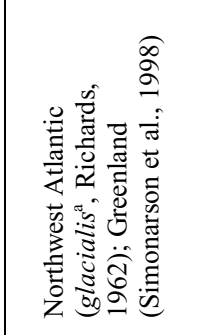 \\
\hline & & 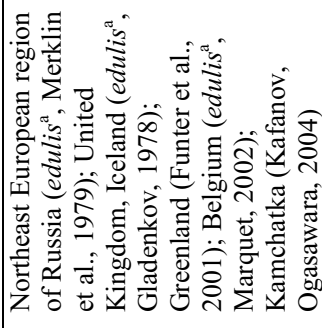 & & 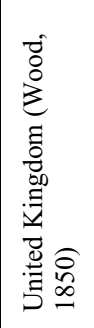 & 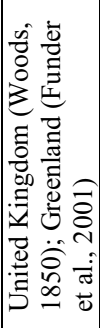 & 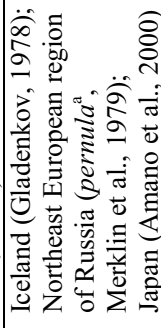 & 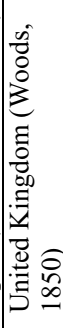 & 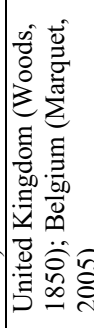 & 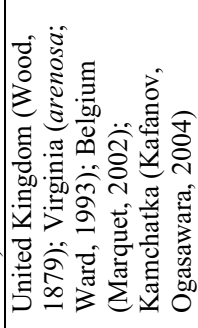 \\
\hline 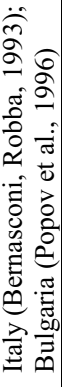 & & 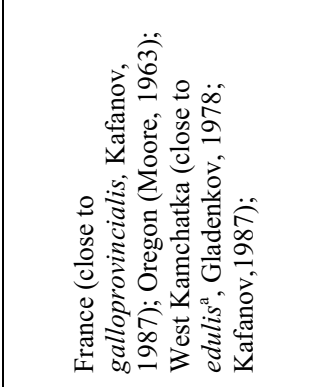 & & & 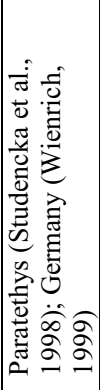 & 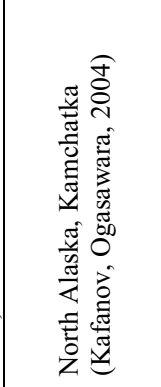 & 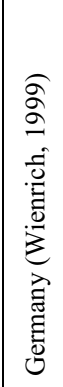 & 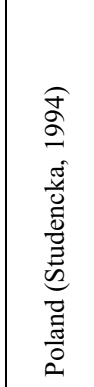 & 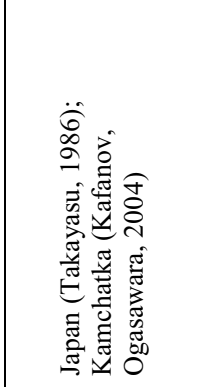 \\
\hline$\stackrel{8}{\Sigma}$ & 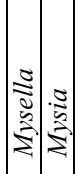 & 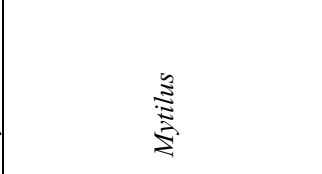 & 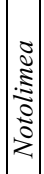 & 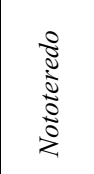 & 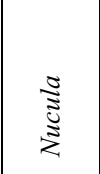 & $\frac{\sqrt[Z]{3}}{\frac{Z}{3}}$ & 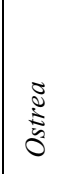 & 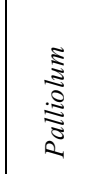 & 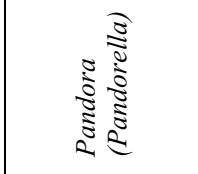 \\
\hline 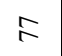 & $\infty 2$ & 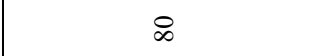 & $\bar{\infty}$ & $\widetilde{\infty}$ & $\infty$ & $\underset{\infty}{\ddagger}$ & 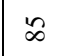 & $\stackrel{\infty}{\circ}$ & $\hat{\infty}$ \\
\hline
\end{tabular}




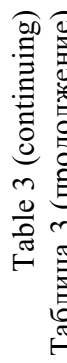

\begin{tabular}{|c|c|c|c|c|c|c|c|c|c|c|c|c|c|c|c|}
\hline 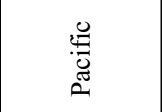 & $\mid$ & 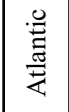 & 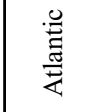 & 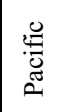 & 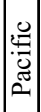 & 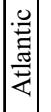 & 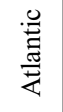 & $\begin{array}{l}\text { 莺 } \\
\text { 吾 }\end{array}$ & 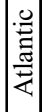 & 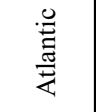 & 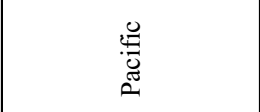 & \begin{tabular}{l|l}
0 \\
$\vdots$ \\
\end{tabular} & 气 & & 总 \\
\hline $\begin{array}{l}\mathscr{U} \\
\stackrel{\tilde{J}}{\tilde{D}} \\
\end{array}$ & $\left|\begin{array}{|c|}\mid \frac{\pi}{\pi} \\
\frac{\pi}{2} \\
0 \\
z \\
\end{array}\right|$ & 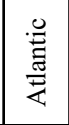 & 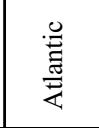 & 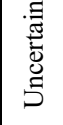 & 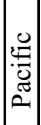 & 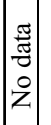 & 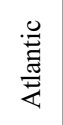 & 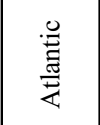 & $\mid$\begin{tabular}{l|}
$\frac{\pi}{3}$ \\
$\frac{\pi}{5}$ \\
0 \\
$z$ \\
$z$
\end{tabular} & $\begin{array}{l}\text { 总 } \\
\text { 恶 }\end{array}$ & $\begin{array}{l}\mathscr{U} \\
\stackrel{\mathscr{J}}{\tilde{E}} \\
\tilde{E}\end{array}$ & 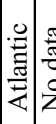 & 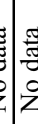 & & $\begin{array}{l}\frac{\pi}{\pi} \\
\frac{\pi}{\pi} \\
0 \\
z\end{array}$ \\
\hline 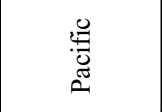 & 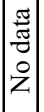 & 总 & 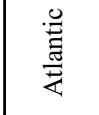 & 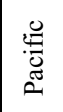 & 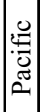 & 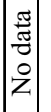 & $\begin{array}{l}\frac{\pi}{\tilde{J}} \\
\text { 苟 } \\
\dot{0}\end{array}$ & 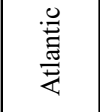 & $\mid$\begin{tabular}{|l}
$\frac{\pi}{\tilde{z}}$ \\
0 \\
0 \\
$z$
\end{tabular} & $\frac{\text { 兽 }}{\text { 严 }}$ & $\begin{array}{l}\mathscr{Z} \\
\mathscr{\Xi} \\
\approx\end{array}$ & 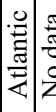 & & & 吾 \\
\hline 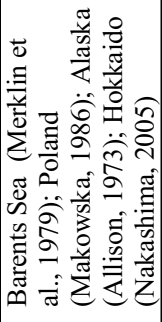 & & 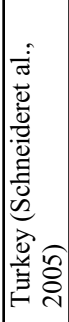 & 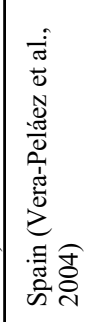 & & & & & & & & 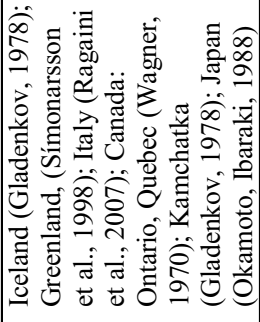 & & & & \\
\hline 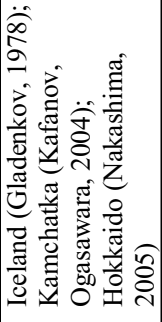 & & 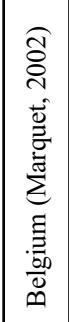 & 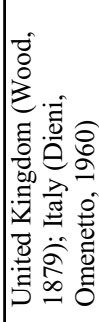 & 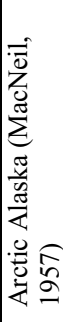 & 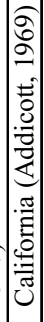 & & 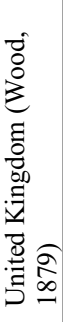 & 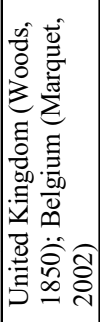 & & 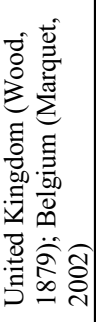 & 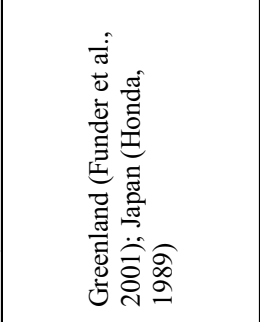 & 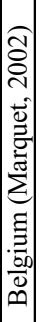 & & & \\
\hline 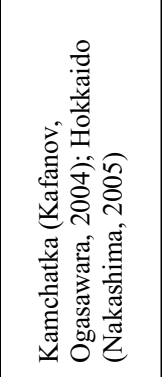 & & 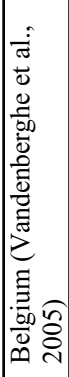 & 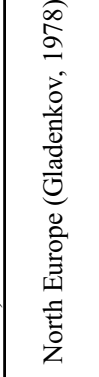 & 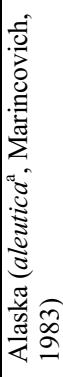 & 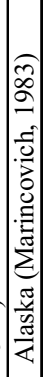 & & & 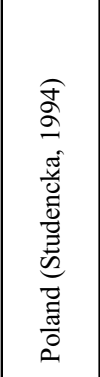 & & 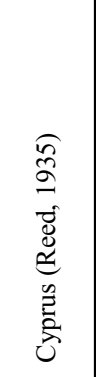 & 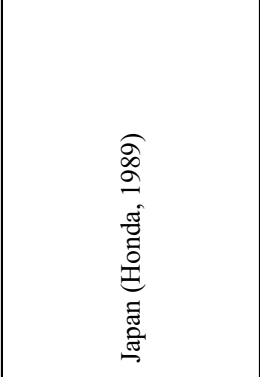 & 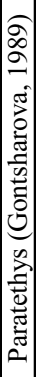 & & & 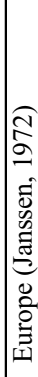 \\
\hline 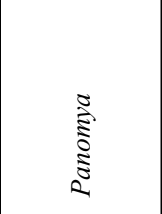 & 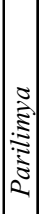 & 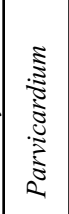 & 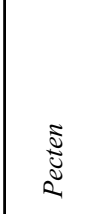 & 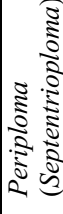 & 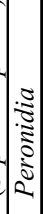 & 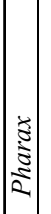 & $\frac{\frac{y}{g}}{2}$ & 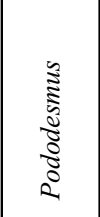 & 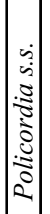 & 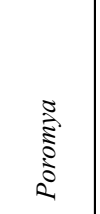 & 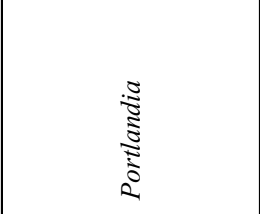 & 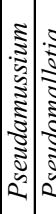 & & & 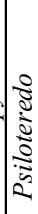 \\
\hline$\infty$ & $\infty$ & \& & $\bar{a}$ & สু & $a$ & ప & $\approx$ & ฉ゚ & ลे & $\stackrel{\infty}{\sigma}$ & à & & $\underline{\underline{e}}$ & & \\
\hline
\end{tabular}




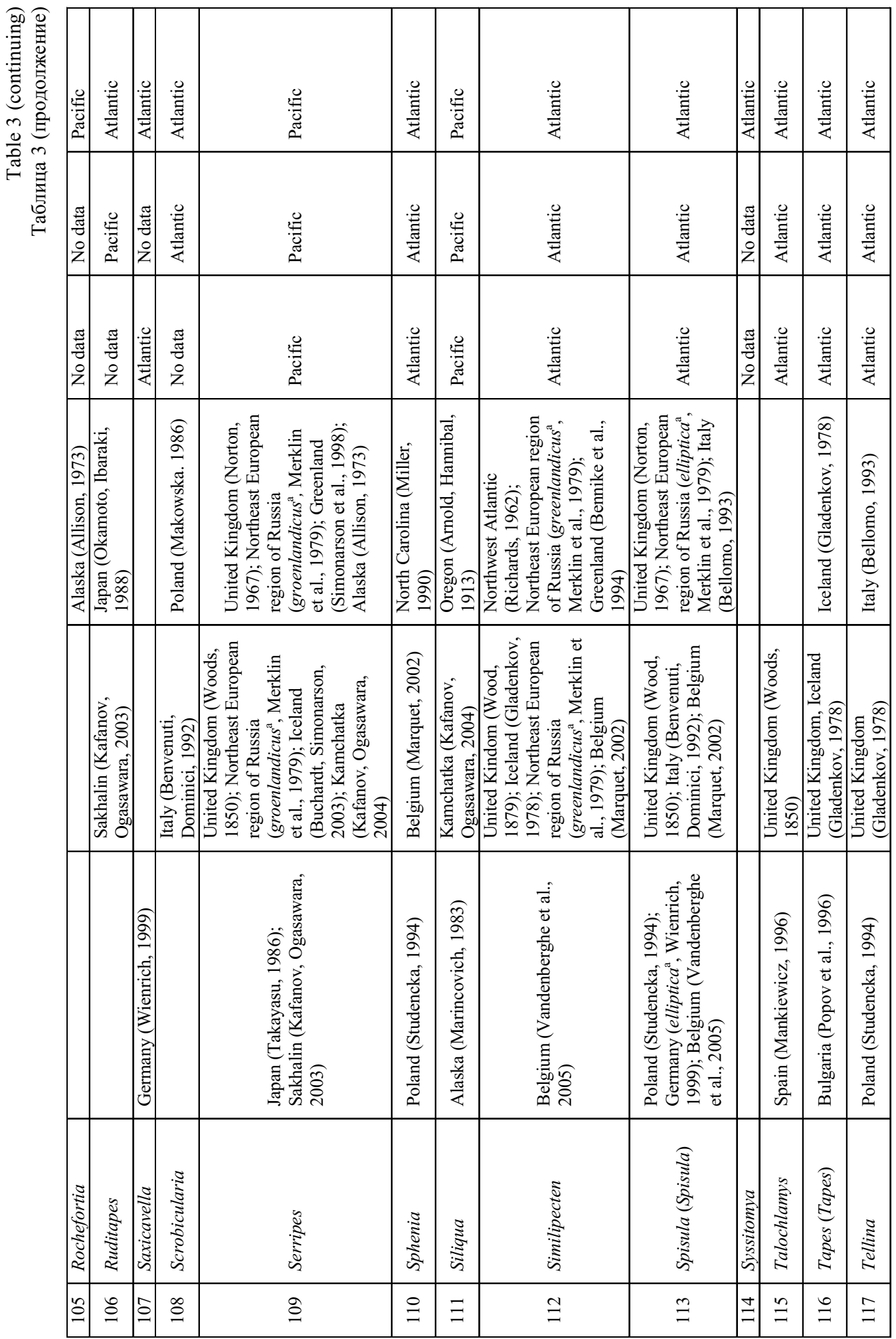




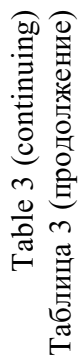

\begin{tabular}{|c|c|c|c|c|c|c|c|c|c|c|c|}
\hline 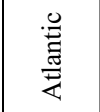 & 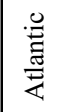 & $\begin{array}{l}\text { 莺 } \\
\text { 严 }\end{array}$ & $\begin{array}{l}\text { 总 } \\
\text { 恶 }\end{array}$ & $\begin{array}{l}\text { 岂 } \\
\text { 尝 }\end{array}$ & 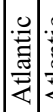 & & 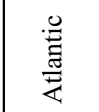 & 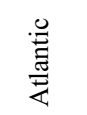 & 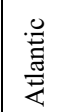 & 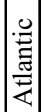 & 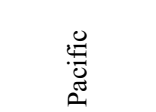 \\
\hline \multirow[t]{2}{*}{$\begin{array}{l}\text { 莺 } \\
\text { 䲶 }\end{array}$} & 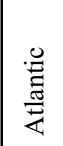 & 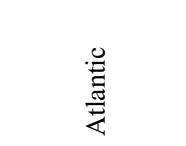 & 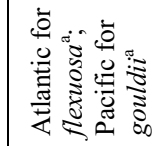 & 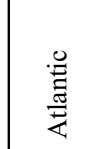 & 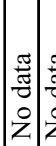 & 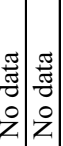 & $\begin{array}{l}\text { 莺 } \\
\text { 严 }\end{array}$ & 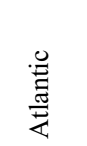 & 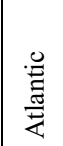 & 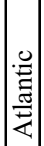 & 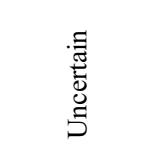 \\
\hline & $\begin{array}{l}\text { 曾 } \\
\text { 焉 }\end{array}$ & 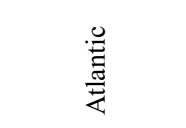 & 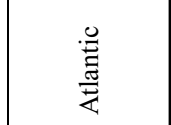 & $\begin{array}{l}\text { 莺 } \\
\text { 焉 }\end{array}$ & 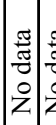 & & 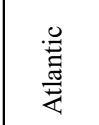 & $\begin{array}{l}\frac{\pi}{\tilde{J}} \\
\text { Z } \\
\text { z }\end{array}$ & 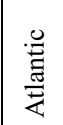 & 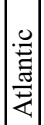 & 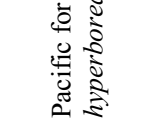 \\
\hline 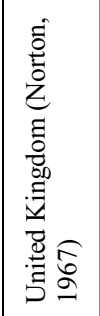 & 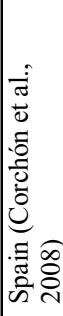 & 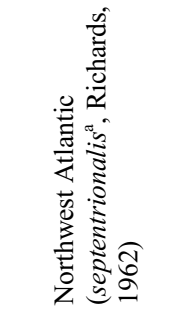 & 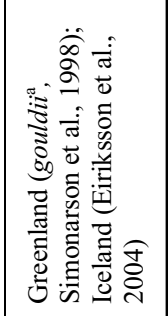 & 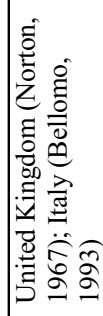 & & 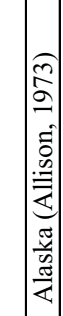 & & & 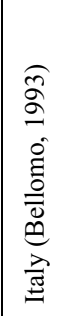 & & 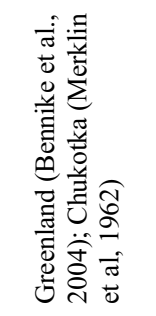 \\
\hline \multirow[t]{2}{*}{ 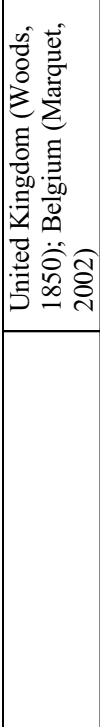 } & 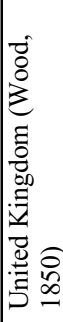 & 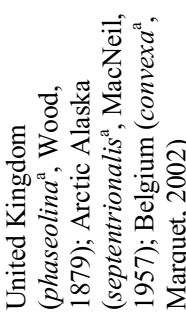 & 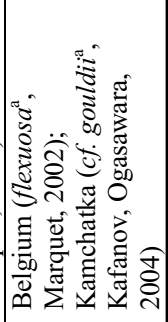 & 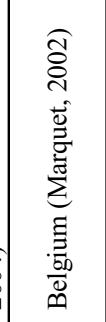 & & & 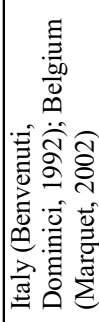 & 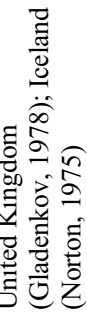 & 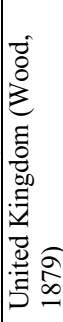 & 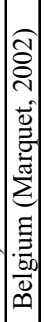 & 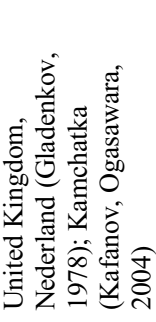 \\
\hline & 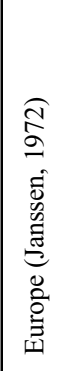 & 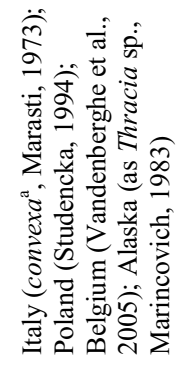 & 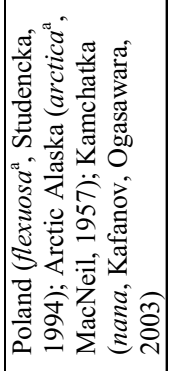 & 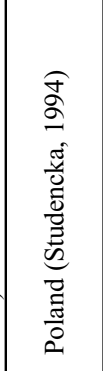 & & & 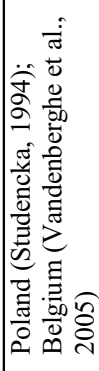 & & 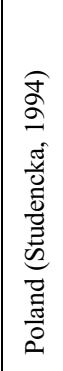 & 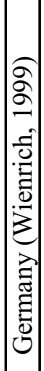 & 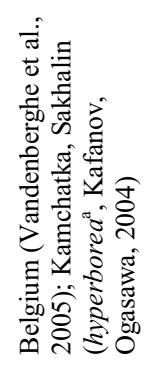 \\
\hline 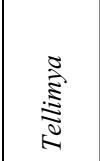 & 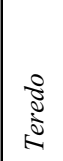 & 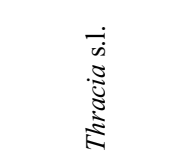 & 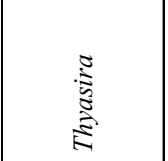 & 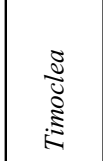 & 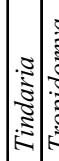 & 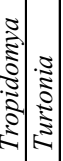 & 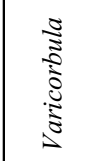 & 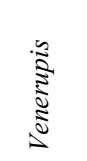 & 气 & 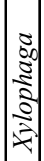 & $\frac{\mathbb{Z}}{d}$ \\
\hline$\stackrel{\infty}{=}$ & $\stackrel{\vartheta}{\exists}$ & 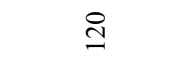 & $\overline{\mathrm{I}}$ & $\tilde{\Xi}$ & $\cong$ & $\stackrel{\Xi}{\Xi} \cong$ & $\underset{\beth}{\beth}$ & $\widehat{\beth}$ & $\stackrel{\infty}{\simeq}$ & $\stackrel{\mathrm{\beth}}{ }$ & 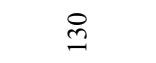 \\
\hline
\end{tabular}


The ratio of species of Atlantic and Pacific origin of bivalve molluscs

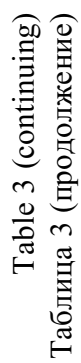

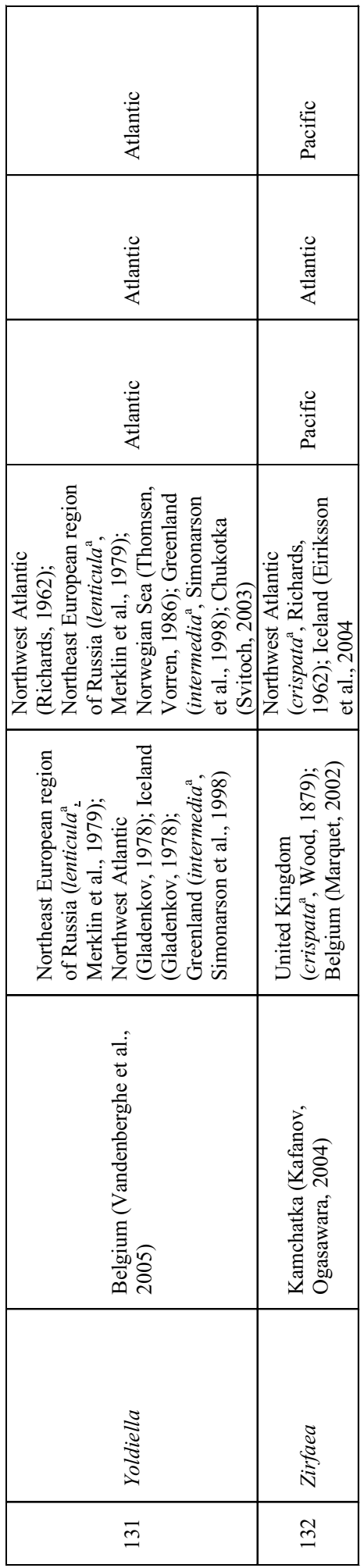

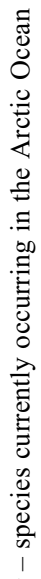


genera in which part of modern Arctic species occur in fossils in the North Atlantic and part in the North Pacific in Late Miocene-Early Pliocene.

The identification of a region, from which penetration into the Arctic took place, by data on the modern distribution of species, was based on a complex of characters of approximately equal importance.

The following distribution patterns were considered as evidence for a higher probability of Pacific origin:

1. A genus is known from the North Pacific and not recorded from the North Atlantic.

2. The number of species is higher in the North Pacific than in the North Atlantic.

3. Arctic species occur also in the North Pacific but are not recorded from the North Atlantic.

4. Arctic species have a near-Pacific distribution.

Analogous criteria were used to assign the origin of species to the Atlantic. Genera were identified as of uncertain origin when evidences for the Pacific and the Atlantic origin were similar. For example, a genus could be represented by more species in the North Pacific, but Arctic species are known from the North Atlantic. Genera were considered to be autochthonous if they are not known outside the Arctic Ocean. These criteria do not allow the determination of the dual colonization of a genus. However, in some cases, for example when there are Arctic species of a genus occurring in the Atlantic and not known in the Pacific, and there are Arctic species occurring in the Pacific but not known in the Atlantic, we suggest independent colonization.

A second analysis was based on the modern distribution of species, in which the presence of species common with the North Pacific was regarded as more important evidence than that with the North Atlantic. So the Pacific origin was adopted for some genera in which the number of North Atlantic species is the same or even higher than North Pacific species (Table 4).

This analysis considers the fact that the probability of penetration of Atlantic species into the North Pacific through the Arctic is less than probability of penetration of species from the North Pacific into the North Atlantic, as evidenced by both paleodata and molecular analysis (Durham, MacNeil, 1967; Vermeij, 1991; Dodson et al., 2007). As s result of both analyses based on the modern distribution of species, we obtained two numbers for genera of supposedly Pacific origin: the first most likely underestimating, the second overestimating the "real" number.

The final conclusion, about the probable region of penetration into the Arctic, was made based on combined data: paleo-records and the pattern of current distribution. Paleo-data was given a higher ranking than the modern distribution. A few available molecular data (for the genera Arctica, Chlamys, Macoma, Modiolus and Mytilus) were used in the analysis (Dahlgren et al., 2000; Galand, Fevolden, 2000; Väinölä, 2003; Nikula et al., 2007; Lockwood et al., 2010).

\section{Results}

Ratio of species in different Arctic regions common with the North Pacific and the North Atlantic

\section{Shallow-water regions}

The number of bivalve species in marginal Eurasian seas (depth up to $300 \mathrm{~m}$ ) diminishes from the Norwegian Sea (193 spp.) towards the East Siberian Sea (59 spp.) and then increases in the Chukchi Sea (72 spp.) (Figs 1, 2) before decreasing in the Beaufort Sea (61 spp.) (Table 2 ). The most drastic changes in species numbers occur at the transition from the Norwegian Sea to the Barents Sea (a nearly a twofold decrease) and from the Barents Sea to the Kara Sea (a 1.5 times decrease). The share of species in common with the Atlantic decreases from the Norwegian to the Chukchi Sea, then it slightly increases in the Beaufort Sea. The trends of changes in the share of species common with the Atlantic and the total species number coincide: the most significant changes occur at the transition from the Norwegian Sea to the Barents Sea and from the latter to the Kara Sea. The share of species common with the Pacific gradually in- 
creases from the Norwegian Sea to the Chukchi Sea, and then decreases in the Beaufort Sea. The most drastic change occurs in transition from the East Siberian Sea to the Chukchi Sea, where the share of species common with the Pacific increases more than twofold. The prevailing share of species common with the Atlantic characterizes the majority of shallow-water regions of the Arctic. The exceptions are the Chukchi and Beaufort seas. The share of species common with both the Atlantic and the Pacific increases from the Norwegian Sea, reaching a maximum in the East Siberian and Beaufort seas. All shallow-water regions, except for the Norwegian Sea, are mainly inhabited by species common to both the Atlantic and the Pacific.

\section{Deep-sea regions}

Deep-sea basins are inhabited by far fewer bivalve species than the shallow-water regions (Fig. 1, 2; Table 2). Deep-sea basins are characterized by the absence, or very small share of, species known only from the Pacific. The Arctic deep-sea basins mainly contain species known either only from the Atlantic (Nansen and Amundsen Basins), or from the Atlantic and also common to both the Atlantic and the Pacific (Norwegian and Greenland Basins), or are Arctic endemics (Canada Basin).

\section{Origin of genera}

\section{Paleontological approach}

Paleo-data was available for 111 genera of Arctic bivalves, but three genera were recorded only from Pleistocene and that is not enough to make conclusions regarding the area of origin. Therefore the paleo-data allowed speculation about origin of 108 genera. Based on paleorecords from Middle Miocene deposits, the area of origin was suggested for 97 genera $(72 \%$ of all Arctic genera) (Figs 6A, 7A). Colonization from the North Atlantic is suggested for 71 genera and from the North Pacific for 23 genera with independent colonization from both regions for three genera (Table 3). Paleo-records dating to the Pliocene were used to reconstruct the area of origin for 99 genera $(74 \%)$. Colonization from the North Atlantic is suggested for 70 genera and from the North Pacific for 17 genera. Independent colonization from both regions is suggested for two genera. The area of origin remains uncertain for 10 genera (Table 3 ).

The large number of genera of uncertain origin in the second version of the analysis can be related to insufficient resolution of the method (too long time periods). For example, if over the entire Pliocene there were records of a genus in both the North Pacific and the Atlantic, the area of the genus origin was considered uncertain. Perhaps the use of the Pliocene subdivisions could help in clarification of the time at which the genus appeared in different regions. The number of genera of the Atlantic origin differs insignificantly between both versions of the analysis.

\section{Neontological approach}

Conclusions about the origin of all 132 genera were based on the four biogeographic criteria presented earlier (Table 4) (Figs 6B, 7B). Based on the first criterion (when a genus is absent in the northern part of one ocean but present in the Arctic and in the north of the other ocean), the region of penetration into the Arctic was identified for 61 genera. The origin of the remaining 72 genera was established in two versions based on the combination of criteria 2-4.

According to the first analysis, with all criteria of equal weight, 98 genera were of Atlantic origin, 21 genera of Pacific origin, one genus was autochthonous and 12 genera were of uncertain origin. In the second analysis, when the Atlantic-Arctic species ranges were considered less important than the Pacific-Arctic species ranges, the ratio between genera of different origin has changed. The group of the Atlantic origin decreased by 12 genera while the group of Pacific origin increased by 15 genera and the group of uncertain origin decreased by 6 genera (Table 4).

Despite the increased number of genera of the Pacific origin in the second analysis, this number was still much lower than the number of genera of the Atlantic origin. The results for 114 genera $(86 \%$ of the Arctic genera) were the same in both versions.

\section{Comparison of results of paleontological and neontological approaches}

The region of penetration into the Arctic was proposed based on both paleontological and neontological data for 104 genera. The results 


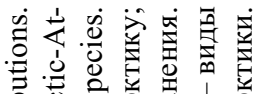

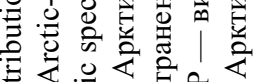

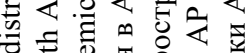

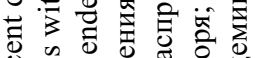
U. के की

난

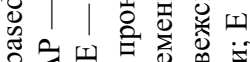

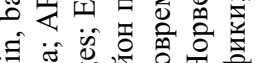
吕 喵. పี $\exists \sum_{0}^{\infty}$

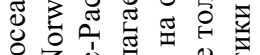
乙号宫志芯

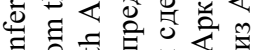
击

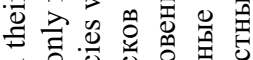
宅.

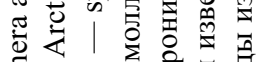

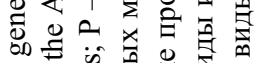

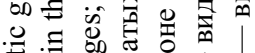

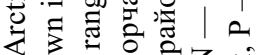

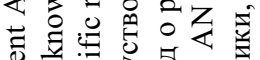
ठु

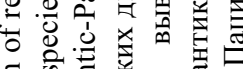

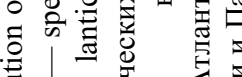
 苟

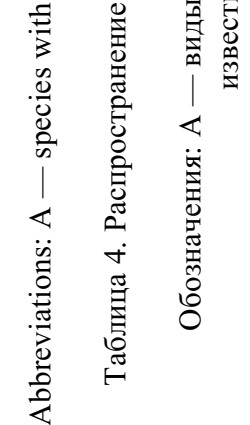




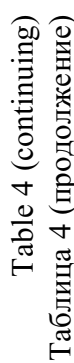

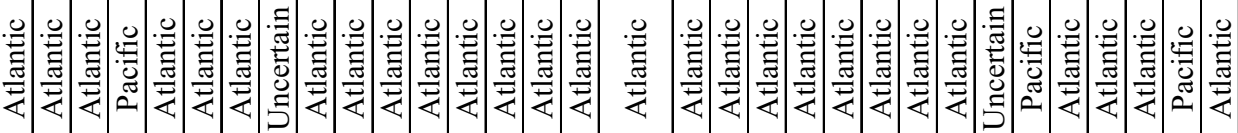

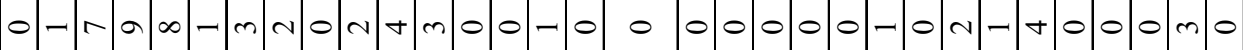

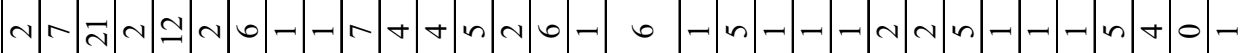

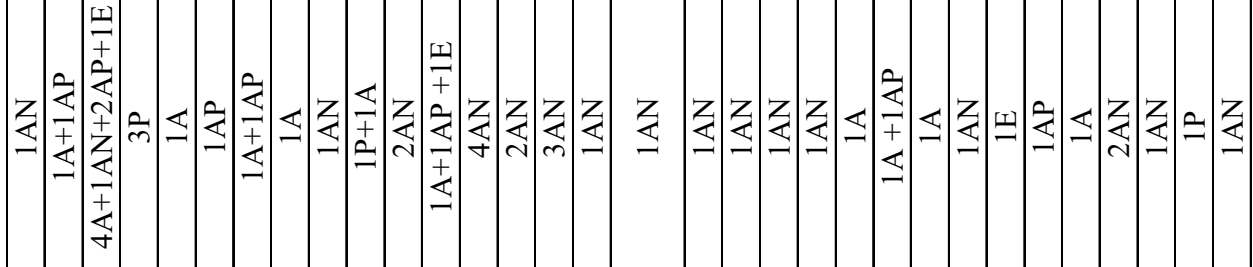

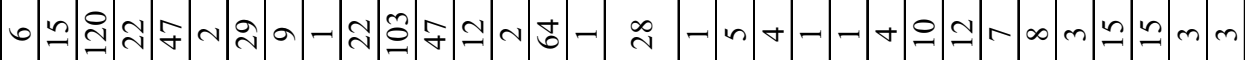

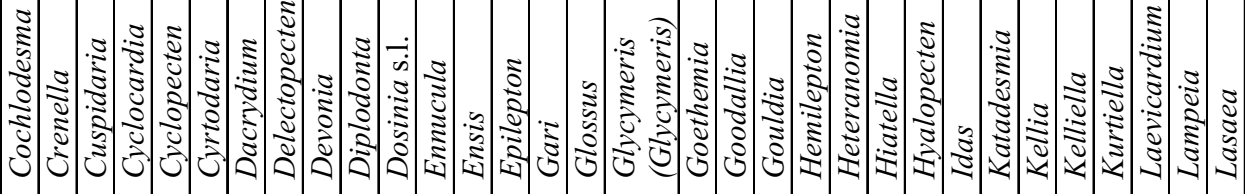

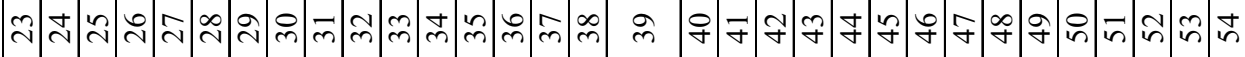




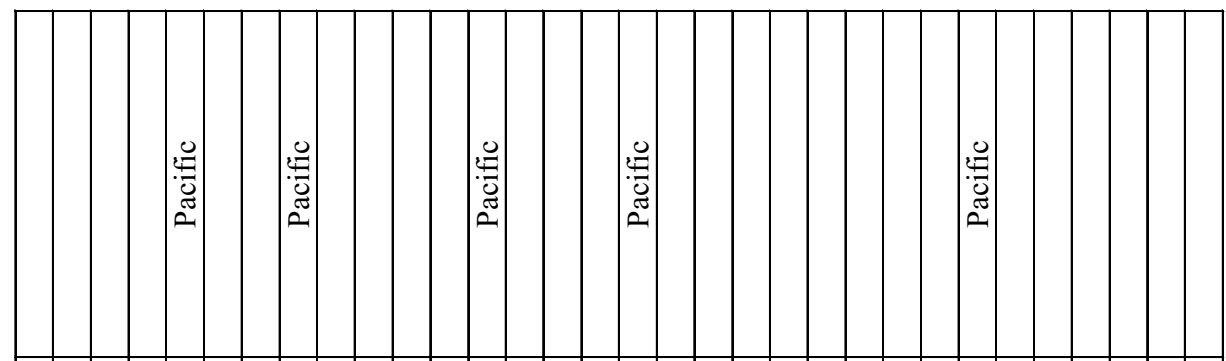

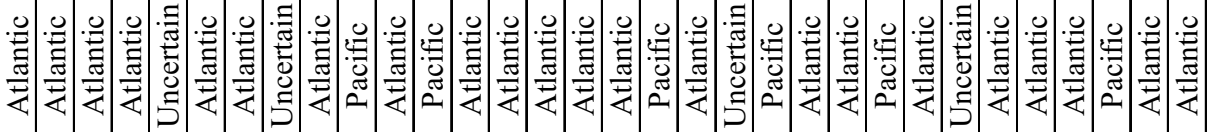

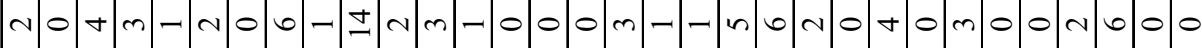

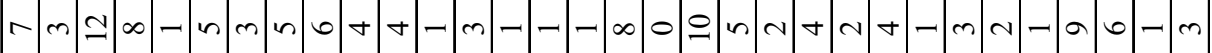

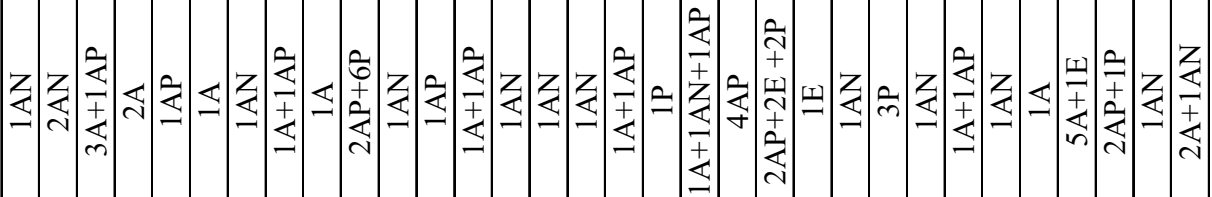

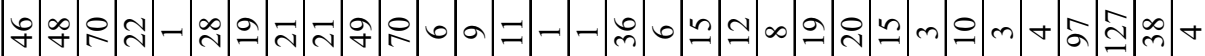

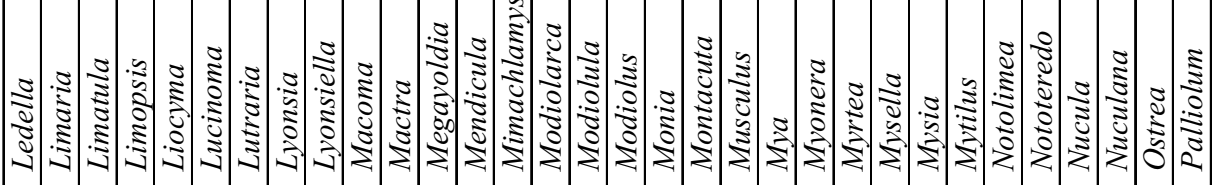

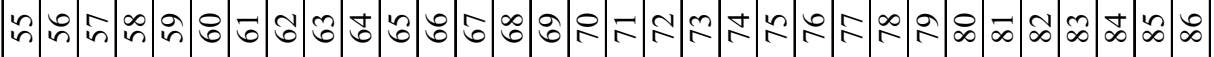


要

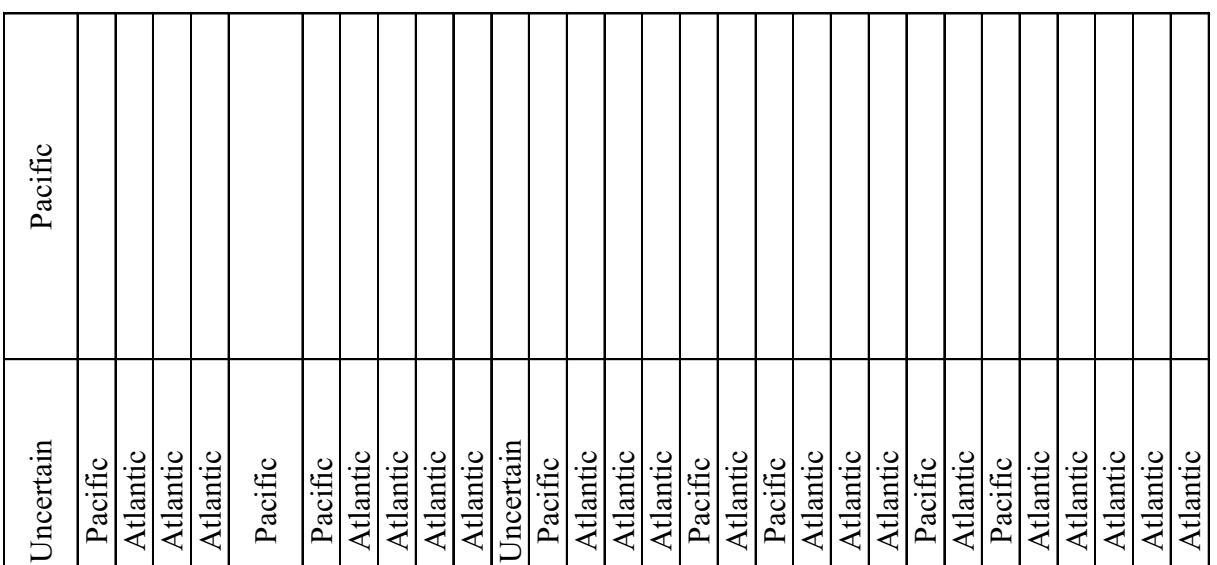

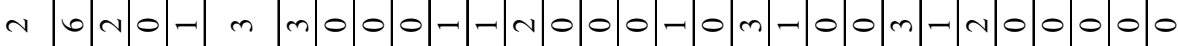

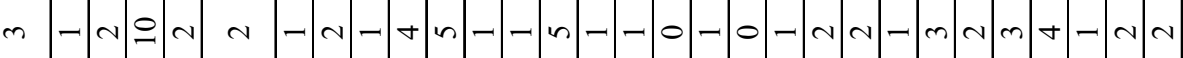

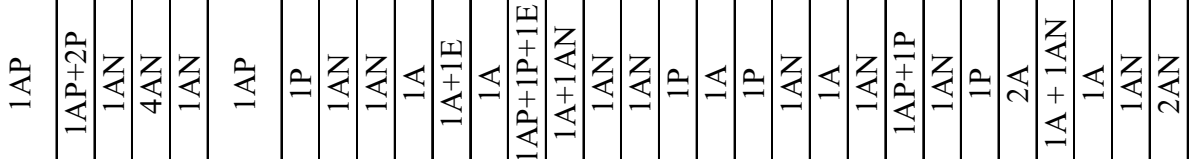

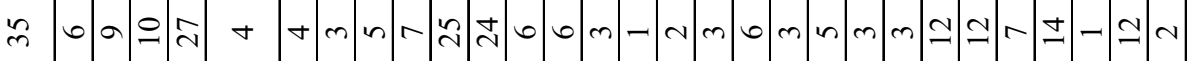

\begin{tabular}{|c|c|c|c|c|c|c|c|c|c|c|c|c|c|c|c|c|c|c|}
\hline 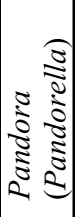 & 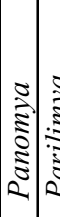 & 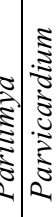 & $\mid \begin{array}{l}\tilde{s} \\
\tilde{d} \\
2 \\
2\end{array}$ & 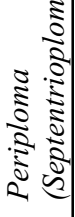 & 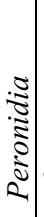 & 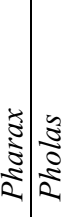 & 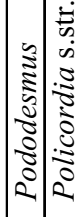 & 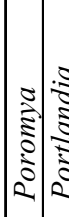 & 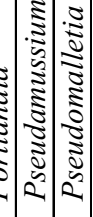 & 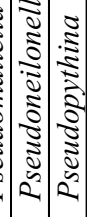 & 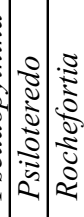 & 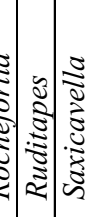 & 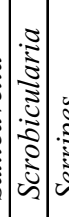 & & $\mid$ & 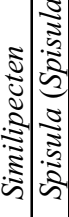 & 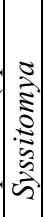 & \\
\hline & & & & 8 & & & & & 0 & & 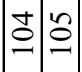 & 8 & $\stackrel{\infty}{0} \stackrel{?}{9}$ & & $\Xi$ & $\cong \stackrel{\oplus}{=}$ & 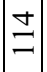 & \\
\hline
\end{tabular}




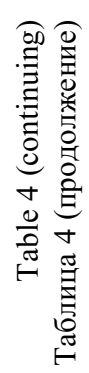

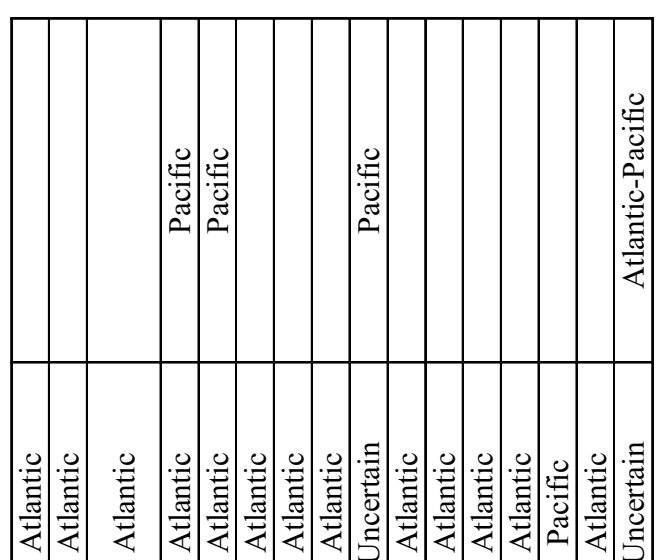

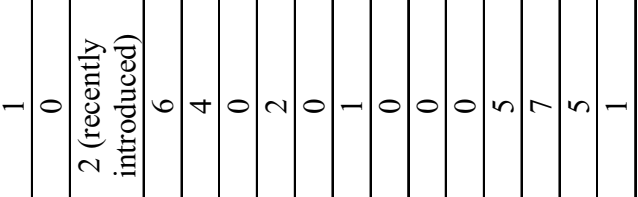

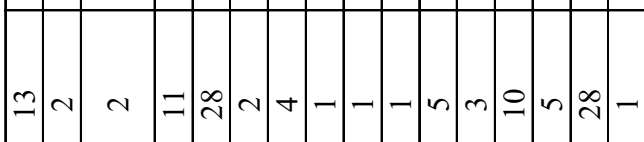

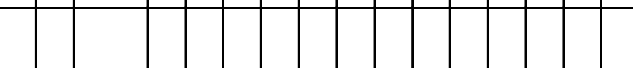

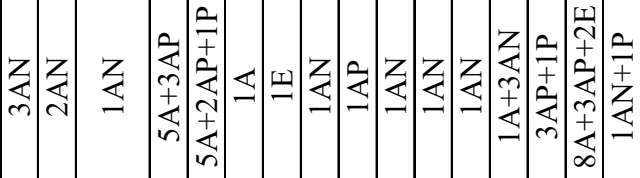

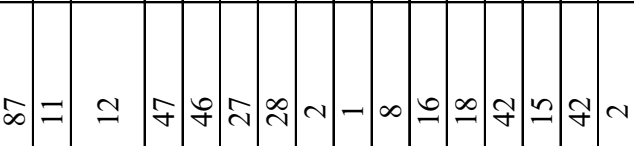

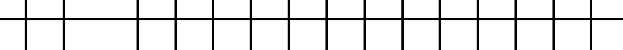

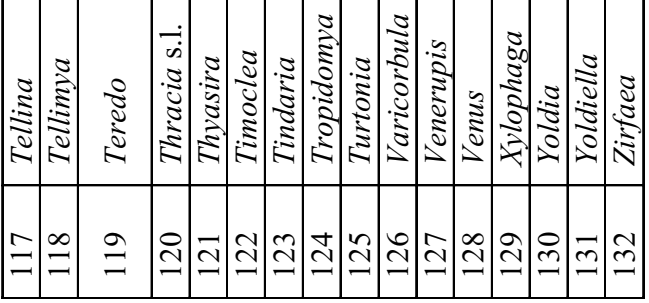

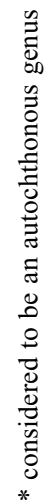


of the two approaches disagreed for 15 genera. The following are several examples where results of neontological and paleontological approaches diverge.

The genus Ennucula according to the formal criterion of the first version of neontological analysis, is of Atlantic origin (Table 4). Since the number of species of this genus occurring in the North Atlantic (four) and the North Pacific (three) differ insignificantly, we consider the ranges of the Arctic species. There are three species of Ennucula in the Arctic. One Arctic species, E. corticata (Møller, 1842), occurs in the near-Atlantic part of the Arctic but not further than the Barents Sea. The second species, E. tenuis (Montagu, 1808), is distributed in both the Atlantic and the Pacific, and the third species, E. romboides (Scarlato, 1981) is an endemic of the Asian Arctic seas. However, in the paleontological records this genus is known from the northern Pacific, Alaska (Merklin et al., 1962) and Oregon (Moore, 1963) since the Middle Miocene. European Miocene deposits lack this genus and, since the Late Pliocene, it appears in Greenland (Funder et al., 2001) and in the Plio-Pleistocene in Iceland (Gladenkov, 1978) (Table 3). Therefore, we suggest that the genus Ennucula colonized the Arctic seas from the Pacific, and the species E. corticata and $E$. romboides apparently are so-called derived species (Vermeij, 1991), i.e. they have formed after the penetration of the genus into the Arctic.

There is only one Arctic species of Ruditapes, R. decussatus (L., 1758). Its range in the Arctic is limited to the Norwegian Sea. In the Atlantic the genus extends to the tropics. Based on the modern distribution of the genus, we suggest that $R$. decussatus colonized the Arctic from the Atlantic. However, the paleo-data do not confirm this: the genus has not been recorded in European deposits. The earliest finding of the genus, is in the Early Pliocene of Sakhalin (Kafanov, Ogasawara, 2003). In the Pleistocene, the genus was recorded in Japan (Okamoto, Ibaraki, 1988). Since the genus is mostly subtropical, we suggest that it penetrated the Atlantic from the North Pacific, having spread along the low latitudes. Eventually, this genus colonized the Arctic from the Atlantic.

Another genus, Modiolus, has two Arctic species, both distributed also in the Atlantic.
One species, M. adriaticus, does not occur in the Arctic beyond the Norwegian Sea and is not known from the Pacific. The other species, $M$. modiolus, occurs in the Norwegian, White and Barents Seas and is known from the Bering Sea. Based on neontological criteria, the genus has penetrated the Arctic from the Atlantic, because there are eight species of this genus in the North Atlantic and only three in the North Pacific. In fossils, the genus is known from the Middle and Late Miocene of Europe (M. adriaticus, Studencka, 1994) as well as Sakhalin (Kafanov, Ogasawara, 2003). Thus, we can only suggest the dual colonization of the Arctic: $M$. adriaticus has Atlantic roots whereas M. modiolus is of the Pacific origin. This "dual-origin" colonization pattern is suggested also for Musculus and Lyonsia.

Based on the modern distribution, it is impossible to establish the region of the Arctic penetration for the genus Mytilus, with two Arctic species, M. edulis and M. trossulus. $\mathrm{Pa}-$ leontological data indicate most likely the $\mathrm{Pa}-$ cific roots for Mytilus. Species similar to $M$. edulis have been recorded in the North-West Pacific since the Early Pliocene (Kafanov, 1987). In the North Atlantic, M. edulis has been recorded since the Early Pliocene in deposits from the United Kingdom, Iceland and Belgium (Durham, MacNeil, 1967; Gladenkov, 1978). There are numerous reconstructions of the biogeographic history of the genus Mytilus based on molecular data, allozyme studies and radiocarbon dates of shells (Varvio et al., 1988; Hansen et al., 2011; Väinölä, Strelkov, 2011). These data suggest that Mytilus migrated from the Pacific to the Atlantic at least twice (Varvio et al., 1988). In the first stage, the Pacific species penetrated the Arctic in the Pliocene. Then, after cooling, colonization pathways closed and the Atlantic and Pacific populations diverged and gave origin to the two species, M. edulis and M. trossulus, respectively. Isolation of the Mediterranean population of $M$. edulis has subsequently led to the origin of $M$. galloprovincialis (Varvio et al., 1988). At the second stage, in the Late Pleistocene or in the Holocene the Pacific species M. trossulus migrated again to the Atlantic through the Arctic.

The comparison of neontological and paleontological results shows that the most efficient criterion in the neontological analysis is the 


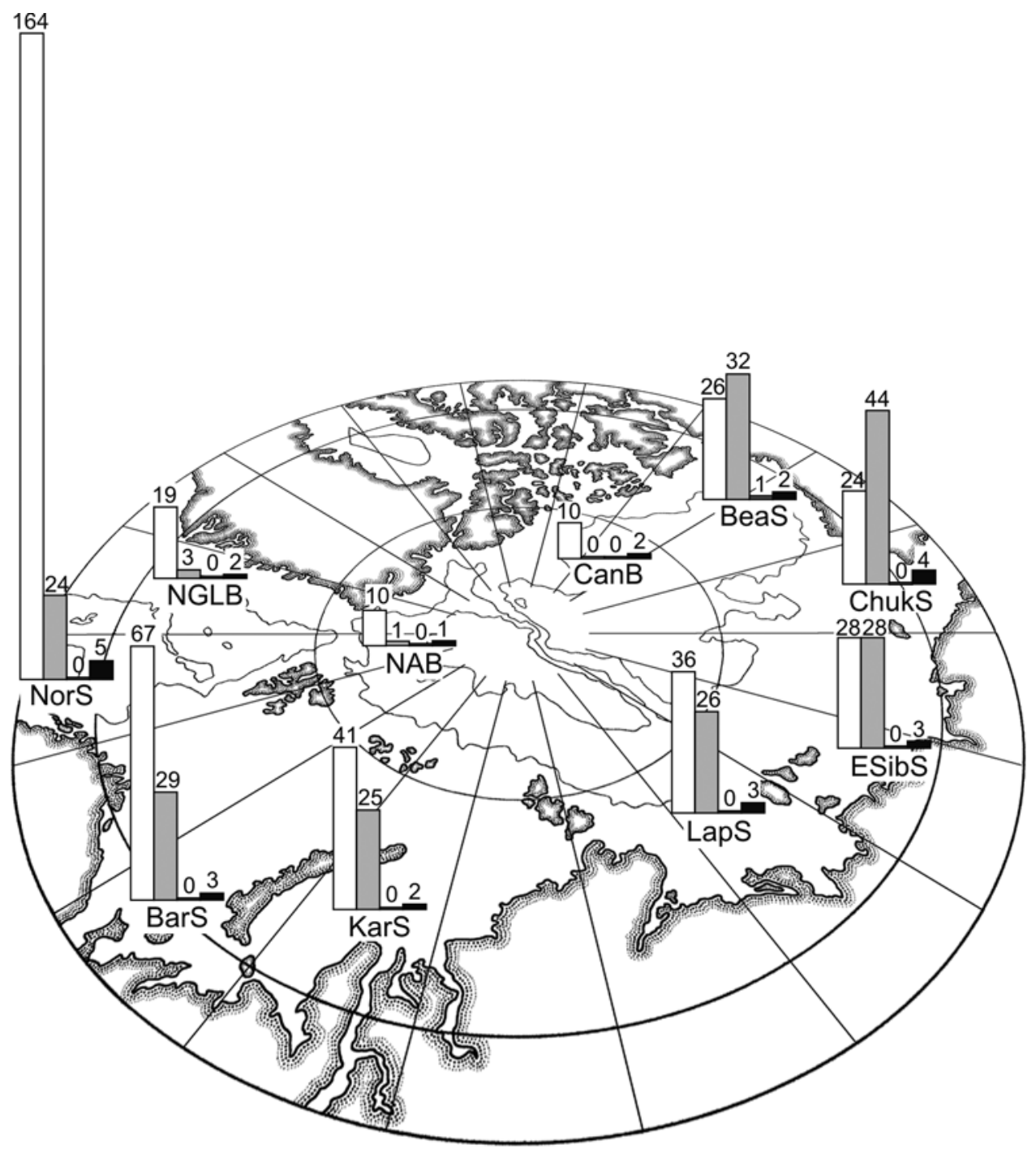

\section{$\square$ Atlantic $\square$ North Pacific $\square$ Autochtonous $\square$ Indet}

Fig. 3. Number of species of Bivalvia of different origin in various Arctic regions.

Abbreviations: see Fig. 1.

Рис. 3. Число видов двустворчатых моллюсков различного происхождения в разных регионах Арктики.

Обозначения те же, что на Рис. 1.

absence of a genus in the near-Arctic region of one ocean and its presence in the Arctic and near-Arctic area of another ocean. Conclusions based on this criterion corresponded to paleodata in all cases. Based on the criterion of the presence of a genus in the northern regions of both oceans, but with more species in one region than in the other, our conclusions agreed with paleo-data in $94 \%$ of cases. Good correspondence of results supports the choice of criteria for the identification of the area of origin based on the modern distribution. This allowed inclusion, into final analysis, of genera without paleontological records. 
In total, among 132 genera, 97 are supposedly of Atlantic origin, 26 of Pacific origin. Three genera are of "dual-origin" and one genus is of autochthonous origin. The origin remains uncertain for five genera (Table 3).

\section{Endemics of the Arctic}

Twenty species of bivalves ( $8 \%$ of the total number) are endemic to the Arctic among the species considered. In shallow-water areas, the share of endemics varies from 0 in the Norwegian Sea to $5 \%$ in the Laptev Sea. In deep-water basins the percentage of endemics is higher: $13 \%$ in the Norwegian and Greenland basins, $33 \%$ - in the Nansen and Amundsen basins and $50 \%$ in the Canada Basin (Figs 1, 2). Among endemic species, 14 belong to genera of Atlantic origin (Nucula zophos, Tindaria derjugini, Yoldiella frami, Y. tamara, Astarte nuuki, A. moerchi, A. elonga, A. neocrassa, A. vaigati, $A$. warhami, Axinulus careyi, Policordia ushakovi, Myonera centobi, Cuspidaria turgida), three belong to genera of Pacific origin (Ennucula romboides, Mya neouddevallensis, M. neoova$t a)$, two species belong to genera of uncertain origin (Katadesmia kolthoffi, Cetomya sp.) and one species belongs to the autochthonous genus (Boreacola vadosus). All endemics of Pacific origin are sublittoral, whereas all endemics of the Atlantic origin, except for the species of Astarte, are the deep-sea species and nearly all occur in the central basins. In the genus Astarte the number of endemic species is exceptionally high: 6 . These species, until recently, have been considered as morphological variations of three widely-spread species (borealis, elliptica and montagui). They received a species rank in the work of Petersen (2001). The autochthonous genus Boreacola is monotypical.

\section{Distribution of species of different origin in Arctic regions}

According to our data, species of genera of Atlantic origin prevail in both the shelf and the deep-sea regions of the Arctic. The share of species of Atlantic origin decreases in marginal Eurasian seas from the Norwegian Sea to the Chukchi Sea and then it somewhat increases in the Beaufort Sea (Figs 3, 4). The share of species of the Atlantic origin decreases most sharply at the transition from the Norwegian Sea to the Barents Sea and from the East Siberian Sea to the Chukchi Sea. The share of species of Pacific origin increases from the Norwegian Sea to the Chukchi Sea and then somewhat declines in the Beaufort Sea. Species of Pacific origin outnumber those of Atlantic origin only in the Chukchi and Beaufort Seas. In the East-Siberian Sea the number of species of different origin is equal.

In all deep-sea regions the share of species of the Atlantic origin is much higher than that of the Pacific origin.

\section{Discussion}

Two different approaches were used in the present study to identify the region of penetration of bivalve species into the Arctic Basin. Both of them, the first based on the modern distribution and the second based on paleo-data, gave similar results. Among 104 genera, the results of the two approaches agreed for 86 genera $(83 \%)$. This high degree of coincidence indicates the reliability of the criteria used.

The results suggest that most of the Recent Arctic bivalves are of Atlantic origin. To test this conclusion, we conducted additional analysis with an enhanced weighting of criteria supporting the Pacific origin. Even in this case, the genera and species of Atlantic origin clearly prevailed.

Our results (Table 2), in principle, agree with data of Fedyakov and Naumov (1987) for the whole Arctic. According to these authors, a minimum of $55 \%$ of species are of Atlantic origin and a minimum of $12 \%$ are of Pacific origin. However, other authors came to different conclusions. Filatova (1957a) agreed that species of Atlantic origin have the larger share, but she also believed that the impact of autochthonous species was very high. According to Kafanov (1979), species of Pacific origin dominate: $70 \%$ compared with $17.5 \%$ of North Atlantic origin and with about $12.5 \%$ of species of unclear origin. Kafanov (1979) explained the similarity of modern faunas of the Arctic and the North Atlantic by the fact that many Arctic species are secondarily Atlantic by origin. Ancestors of such species migrated from the North 


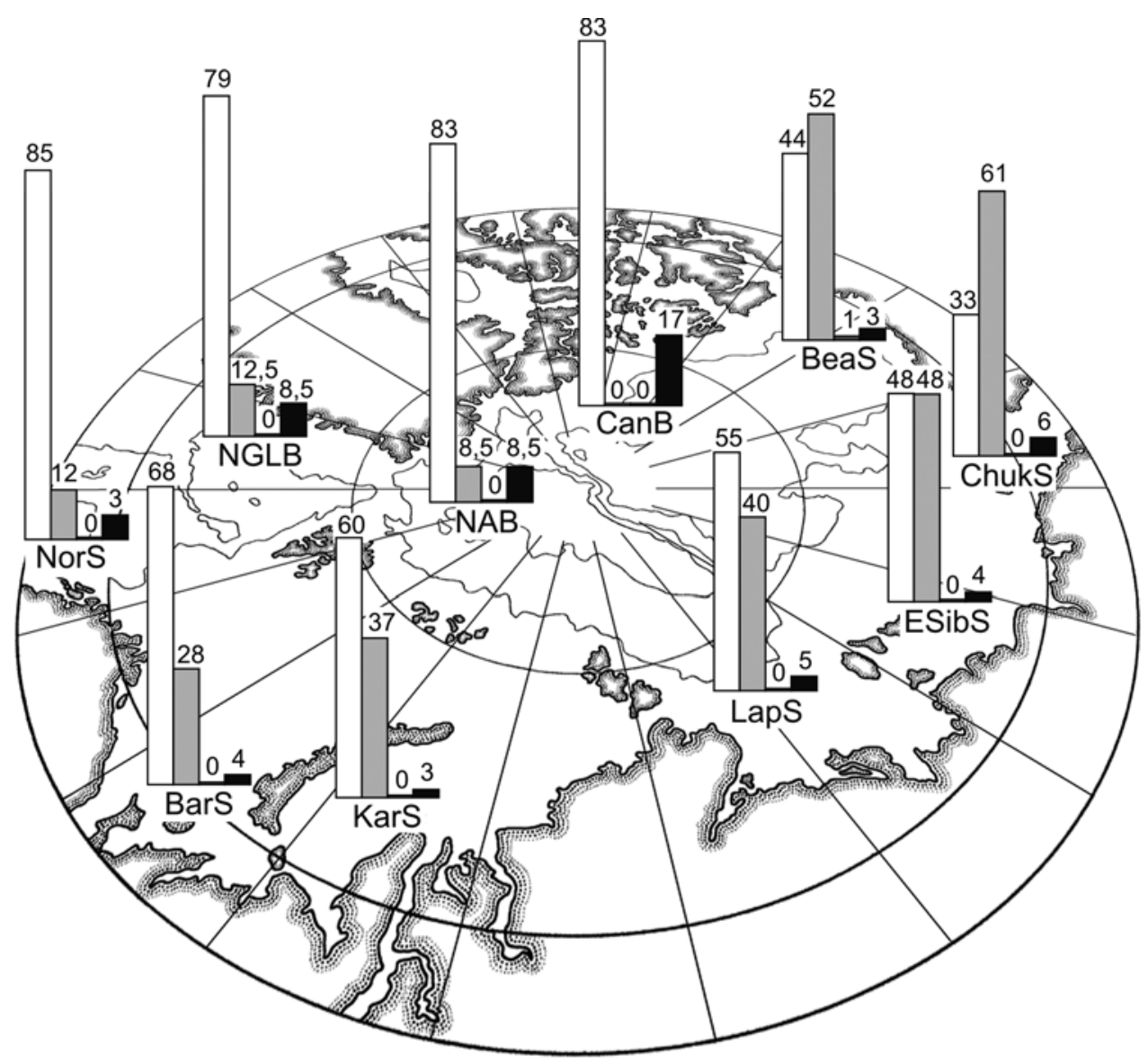

\section{Atlantic $\square$ North Pacific $\square$ Autochtonous $\square$ Indet}

Fig. 4. Percentage of Bivalvia species of different origin in various Arctic regions.

Abbreviations: see Fig. 1.

Рис. 4. Процентное соотношение видов двустворчатых моллюсков различного происхождения в разных регионах Арктики.

Обозначения те же, что на Рис. 1.

Pacific through the Arctic to the North Atlantic and then, during periods of climate warming, these species or their derivatives colonized the Arctic from the North Atlantic.

These discrepancies between the results of different authors may in part be explained by differences in the methods used. For example, Filatova (1957a) did not include the Norwegian Sea in the analysis, thus she excluded a number of species of Atlantic origin. In addition we assigned many of Filatova's autochthonous Arctic species to genera of Atlantic or Pacific origin, depending on the biogeographical history of a genus (see Materials and Methods). According to our approach, only species of endemic Arctic genera were considered as truly autochthonous. Unfortunately, we cannot compare our methods with the approach of Kafanov (1979) since he did not describe it in sufficient detail.

The present results differ from those for shelf starfish. In the Arctic Asteroidea, the prevalence of taxa of Pacific origin was shown by Djakonov (1945) and Mironov, Dilman (2010). Mironov and Dilman (2010) used the same criteria as used in the present study to identify 


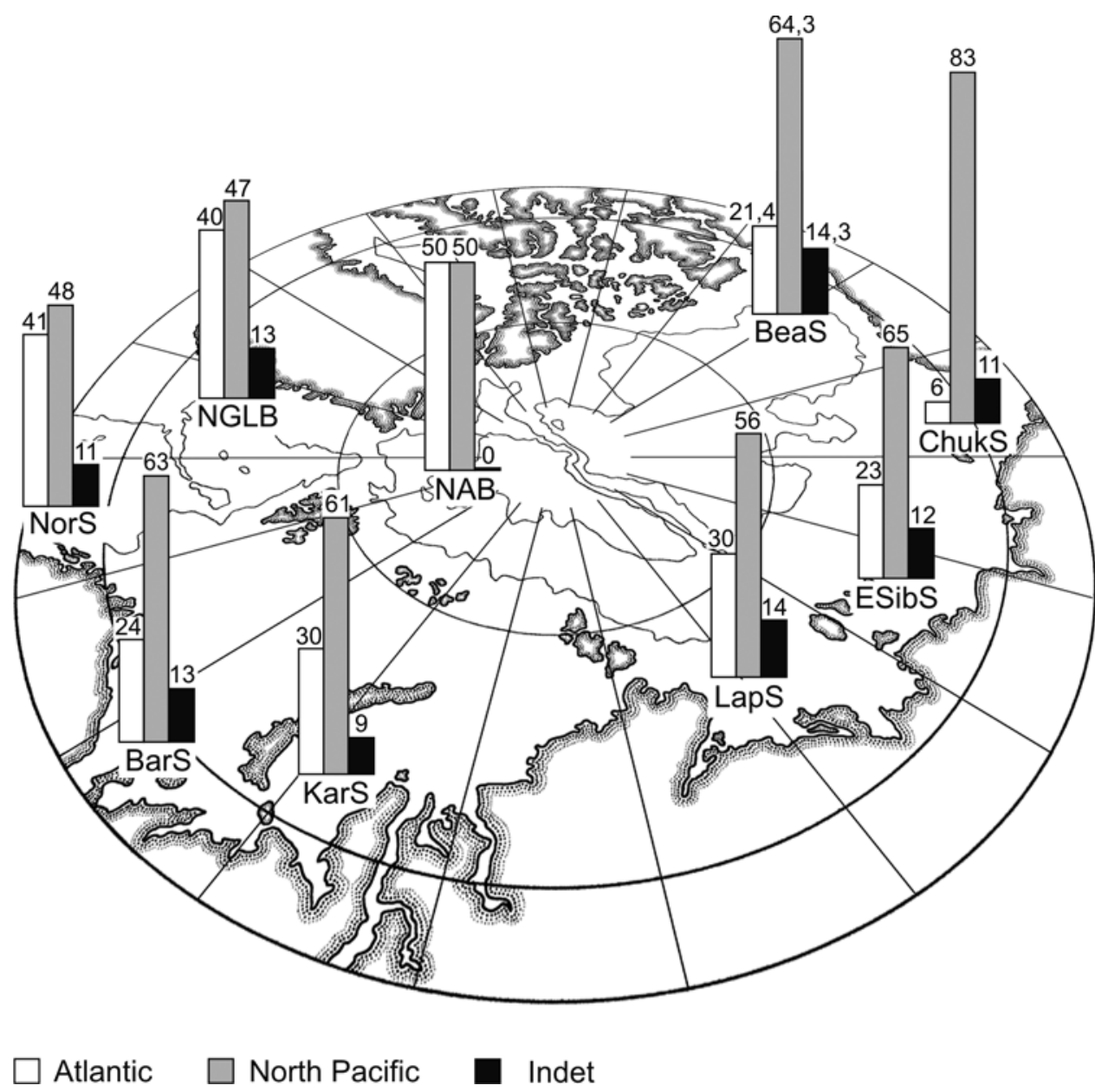

Fig. 5. Percentage of species of Asteroidea of different origin in various Arctic regions (after Mironov, Dilman, 2010).

Abbreviations: see Fig. 1.

Рис. 5. Процентное соотношение видов морских звезд различного происхождения в разных регионах Арктики.

Обозначения те же, что на Рис. 1.

the origin of genera (Fig. 5). Thus, different proportions of genera of the Atlantic and the Pacific origin are most likely taxon-specific and reflect the different biogeographic history of bivalve molluscs and sea stars in the Arctic. Differences in the patterns of modern regional distribution of bivalves and starfish in the Northern Hemisphere support such assumption. For example, the share of genera recorded in the North Atlantic and in the Arctic, but unknown from the North Pacific, is higher in bivalves than in sea stars: 45 and $31 \%$ respectively. Genera of sea stars unknown from the North Pacific are characterized by a low number of species in the North Atlantic: usually 1-3 (5 species only in the case of Poraniomorpha). Genera of bivalves unknown from the North Pacific often contain more than three species in the North Atlantic, the maximum being 10 species in Parvicardium.

The different biogeographic history is apparently associated with a different evolutionary plasticity in the two taxa. The evolutionary plasticity of Bivalvia is higher than of Asteroidea. The higher number of species, in bivalve 


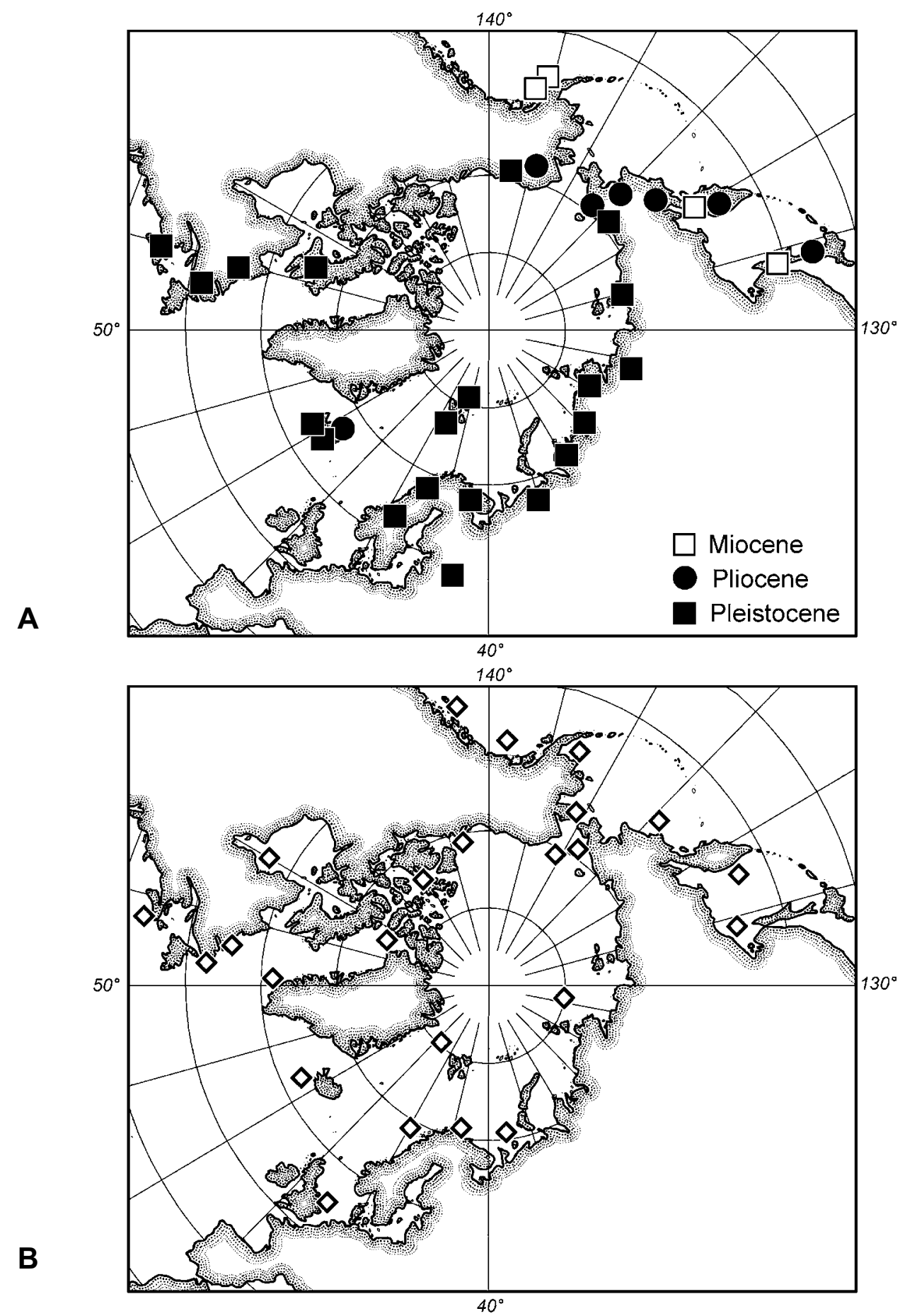

Fig. 6. Paleo-records and recent distribution of the genus Ciliatocardium Kafanov, 1974. A - paleo-records (after Kafanov, 1980; Kafanov et al., 2001); B - recent distribution in the Arctic.

Рис. 6. Палео- и современное распространение двустворчатых моллюсков рода Ciliatocardium Kafanov, 1974.

А — палео-нахождения (по Kafanov, 1980; Kafanov et al., 2001); В — современное распространение в Арктике. 


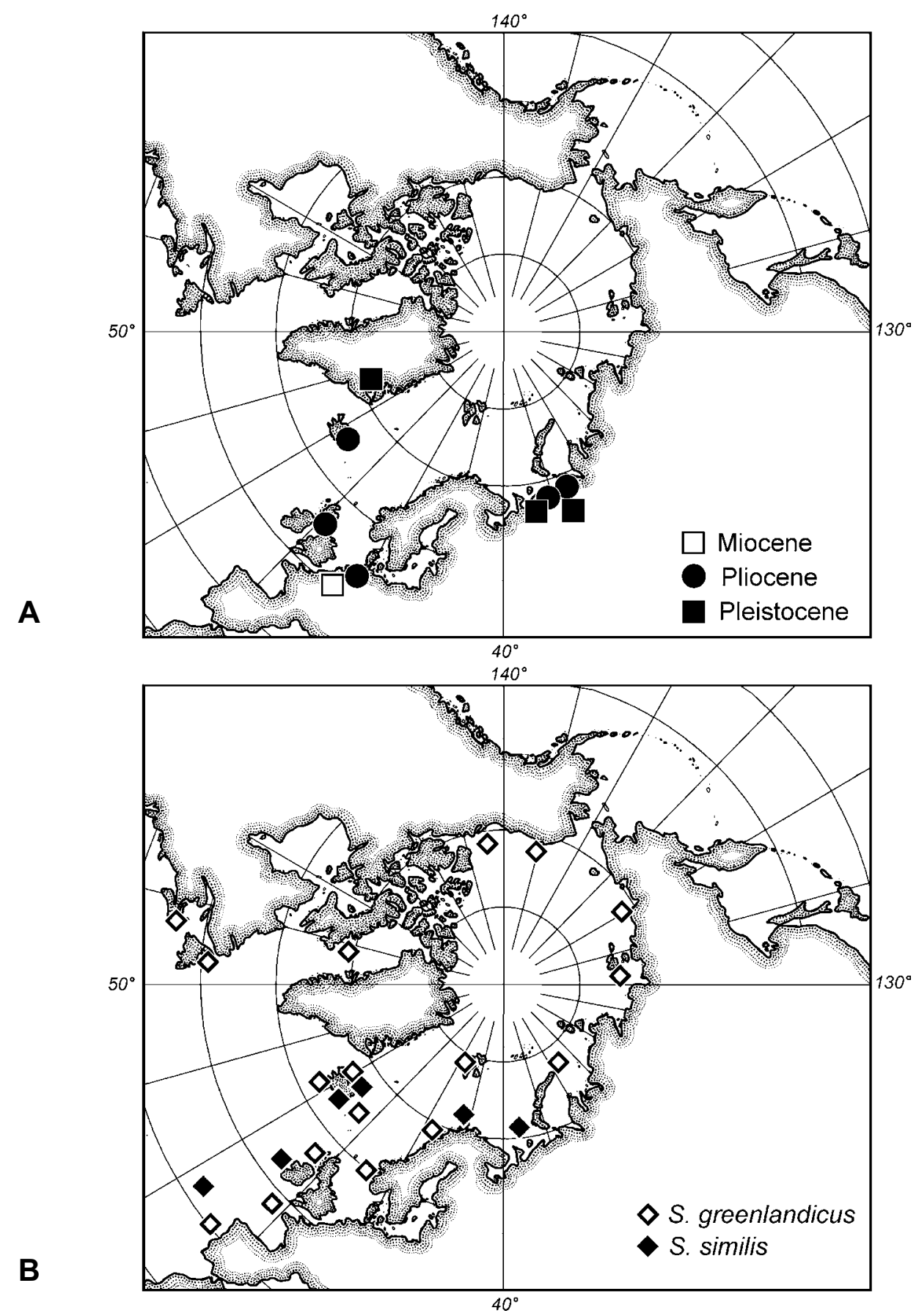

Fig. 7. Paleo-records and recent distribution of the genus Similipecten Winckworth, 1932.

A - paleo-records (after Gladenkov, 1978; Merklin et al., 1979; Marquet, 2002); B - recent distribution in the Arctic. Рис. 7. Палео- и современное распространение двустворчатых моллюсков рода Similipecten Winckworth, 1932.

А - палео-нахождения (по Гладенков, 1978; Мерклин и др., 1979; Marquet, 2002); В - современное распространение в Арктике. 
molluscs as a whole, shows this. During periods of glaciation, the most pronounced changes in the marine environment occurred in the North Atlantic and in the near-Atlantic Arctic. Evolutionary flexible groups, such as bivalves, would be expected to be more successful in periods of rapidly changing environment and quicker in restoring their diversity.

According to numerous published data, the trans-Arctic exchange between faunas of the North Atlantic and the North Pacific was asymmetrical: the number of colonists from the $\mathrm{Pa}$ cific was higher than the number of colonists from the Atlantic (Andriyashev, 1939; Durham, MacNeil, 1967; Kafanov, 1979; Vermeij, 1991). Possible reasons for this asymmetry were discussed by Vermeij (1991). Our data on Recent Arctic bivalves confirm this asymmetry of transArctic migration. The number of genera having crossed the Arctic from the Pacific is 1.4 times higher than that from the Atlantic. The fact of prevalence of species of Atlantic origin in the Arctic, along with higher share of genera of Pacific origin in the trans-Arctic migration requires explanation. Most of the genera of the Pacific origin that crossed the Bering Strait $(69 \%)$ penetrated also the North Atlantic. On the contrary, only $14 \%$ of the Arctic genera of the Atlantic origin occur also in the North Pacific. Thus, most taxa that penetrated the Arctic from the North Pacific participated in the transArctic exchange, whereas most taxa of the Atlantic origin remained within the Arctic Ocean.

These differences in dispersal patterns of taxa of the Atlantic and the Pacific origin might be related to features (nature) of barriers for dispersal. It can be suggested that the boundary between the Arctic and the Atlantic can be easier penetrated in both directions than the Bering Strait. The area of contact of the Arctic and the Atlantic faunas is much wider than the Arctic-Pacific zone of contact and also there are various deep-water straits between the North Atlantic and the Arctic. The connection between the Arctic and the Pacific is narrow and shallow-water, thus more effective as a barrier. The Bering Strait as a barrier is asymmetric, colonization from the North Pacific to the Arctic is more intensive than from the Arctic to the North Pacific. The prevailing northward trend of colonization from the North Pacific could have two reasons. According to Briggs (1974), in interaction of two faunas colonization occurs mainly from an area with higher diversity (in our case the North Pacific) towards an area with lower diversity. Second, the Bering Strait was opening in periods of transgression and climate warming which also determined the prevailing northward dispersal.

According to our results the majority of deep-sea species belong to genera of Atlantic origin. This is true for all the three studied deepsea regions. Among shallow-water regions, only in the Norwegian Sea is the share of species of Atlantic origin higher. A characteristic feature of the Arctic deep-sea basins is the relatively high number of endemic species in genera of Atlantic origin.

\section{Acknowledgement}

We are deeply grateful to R. Janssen (Senckenberg Forschungsinstitut und Naturmuseum, Frankfurt a. M.) for the permission to use his database on bivalve molluscs of European Neogene. This work is part of the project "Arctic marine fauna: data accumulated in Russia" (subproject of the CoML "Arctic Ocean Diversity" program).

\section{References}

Addicott W.O. 1969. Late Pliocene mollusks from San Francisco Peninsula, California, and their paleogeographic significance// Proc.Cal. Acad. of Sci. Vol.37. No.3. P.57-93.

Allison R.C. 1973. Marine paleoclimatology and paleoecology of a Pleistocene invertebrate fauna from Amchitka Island, Aleutian Islands, Alaska // Palaeogeogr. Palaeoclimatol. Palaeoecol. Vol.13. P.15-48.

Amano K. 1986. Age and characteristics of the so-called "Atsunai-Togeshita fauna" in Hokkaido // Paleo. Soc. of Japan Spec. Papers. Vol.29. P.187-198.

Amano K., Suzuki M., Sato T. 2000. Warm-water influx into Japan Sea in the middle Pliocene - Molluscan faunas from the Tentokuji Formation around Mt. Taihei in Akita Prefecture // J. of the Geol. Soc. of Japan. Vol.106. No.4. P.299-30.

Andriyashev A.P. 1939. [Essays on zoogeography and origin of Ichthyofauna of the Bering Sea and adjacent waters]. Leningrad: Leningr. Gos. Univ. 187 p. [in Russian].

Anisimova N.A. 1986. [Phylum Echinodermata] // G.G. Matishov (ed.). Zhizn' i usloviya ee sushestvovaniya $\mathrm{v}$ benticheskoi zone Barenceva moray. Apatity: Kolskii Branch of Academy of Science of USSR. P.147153 [in Russian].

Appeltans W., Bouchet P., Boxshall G.A., De Broyer C., de Voogd N.J., Gordon D.P., Hoeksema B.W., Horton T., Kennedy M., Mees J., Poore G.C.B., Read G., Stöhr S., Walter T.C., Costello M.J. (eds.). 2012. World Register of Marine Species. Accessed at http:// www.marinespecies.org on 2013-06-03. 
Arnold R., Hannibal H. 1913. The marine Tertiary stratigraphy of the North Pacific coast of America // Proc. American Phil. Soc. Vol.52. No.212. P.559-605.

Balson P.S. 1990. The Neogene of East Anglia - a field excursion report // Tertiary Research. Vol.11. No.2-4. P.179-189.

Bellomo E. 1993. Presenza di “Ospiti boreali” (Mollusca, Gastropoda) nel Pleistocene di Contrada Case Alte (Villa S. Giovanni) // Bol. della Soc. Paleo. Italiana. Vol.32. No.3. P.393-399.

Bennike O., Hansen K.B., Knudsen K.L., Penney D.N., Rasmussen K.L. 1994. Quaternary marine stratigraphy and geochronology in central West Greenland // Boreas. Vol.23. P.194-215.

Benvenuti M., Dominici S. 1992. Facies analysis, paleoecology and sequence stratigraphy in a Pliocene siliciclastic succession, Sanb Miniato (Pisa, Italy) // Bol. della Soc. Paleo. Italiana. Vol.31. No.2. P.241-259.

Bernard F.R. 1979. Bivalve mollusks of the western Beaufort Sea // Contributios in Science, Nat. Hist. Mus. Los Ang. County. Vol.313. P.1-80.

Bernasconi M.P., Robba E. 1993. Molluscan palaeoecology and sedimentological features: an integrated appraoch from the Miocene Meduna section, northern Italy // Palaeogeogr. Palaeoclimatol. Palaeoecol. Vol.100. P.267-290.

Brattegard T., Holthe T. 1997. Distribution of marine, benthic macro-organisms in Norway. Utredning for Direktoratet for naturforvaltning. Trondheim. $380 \mathrm{p}$.

Bouchet P., Warén A. 1979. The abyssal molluscan fauna of the Norwegian Sea and its relation to other faunas // Sarsia. Vol.64. P.211-243.

Briggs J.C. 1974. Marine Zoogeography. New York: McGraw-Hill Book Co. 475 p.

Briggs J.C. 1995. Global Biogeography. Amsterdam: Elsevier. $452 \mathrm{p}$.

Buchardt B., Simonarson L.A. 2003. Isotope palaeotemperatures from the Tjornes Beds in Iceland; evidence of Pliocene cooling. Palaeogeogr. Palaeoclimatol. Palaeoecol. Vol.189. No.1-2. P.71-95.

Coan E.V., Scott P.V., Bernard F.R. 2000. Bivalve seashells of western North America. Marine Bivalve Mollusks from Arctic Alaska to Baja California. Santa Barbara, California, Santa Barbara Museum of Natural History. 764 p.

Corchón M.S., Mateos A., Fernández E.A., Peñalver E., Delclòs X., Made J., van der. 2008. Ressources complémentaires et mobilité dans le Magdalénien cantabrique. Nouvelles données sur les mammifères marins, les crustacés, les mollusques et les roches organogènes de la Grotte de Las Caldas (Asturies, Espagne) // L'anthropologie. Vol.112. P.284-327.

Dahlgren T.G., Weinberg J.R., Halanych K.M. 2000. Phylogeography of the ocean quahog (Arctica islandica): influences of paleoclimate on genetic diversity and species range // Mar. Biol. Vol.137. P.487-495.

Dermitzakis M., Georgiades-Dikeoulia E. 1987. Biozonation of the Neogene invertebrate megafauna of the Hellenic area // Ann. Inst. Geol. Publ. Hung. Vol.70. P.125-136.

Dieni I., Omenetto P. 1960. Studio di una macrofauna del Pliocene inferiore di Orosei (Sardegna) // Riv. Ital. Paleont. Strat. Vol.66. No.4. P.605-618.

Dilman A.B. 2009. [Biogeography of the asteroids of the North Atlantic and Arctic]. Ph. D. Thesis. Moscow:
Institute of oceanology of the Russian Acad. Sci. 214 p. [in Russian].

Djakonov A.M. 1945. [Relationships of the Arctic and Northern Pacific marine faunas on the base of an example of zoogeographical analysis of echinoderms] // Zhurnal obshchey biologii. Vol.4. No.2. P.125-153 [in Russian].

Dodson J.J, Tremblay S., Colombani F., Carscadden J.E., Lecomte F. 2007. Trans-Arctic dispersals and the evolution of a circumpolar marine fish species complex, the capelin (Mallotus villosus) // Mol. Ecol. Vol.16. P.5030-5043.

Durham J.W., MacNeil F.S. 1967. Cenozoic migrations of marine invertebrates through the Bering Strait region // D.M. Hopkins (ed.). The Bering Land Bridge. Stanford, California: Stanford University Press. P.326-349.

Ekman S. 1953. Zoogeography of the sea. London: Sidgwick and Jackson. 417 p.

Eiriksson J., Knudsen K.L., Simonarson L.A. 2004. Lateglacial oceanographic conditions off Southwest Iceland inferred from shallow-marine deposits in Reykjavik and Seltjarnarnes Peninsula // Boreas. Vol.33. P.269-283.

Fedyakov V.V., Naumov A.D. 1987. [Arctic bivalve molluscs] // Priroda. No.3. P.49-57 [in Russian].

Fedyakov V.V., Naumov A.D. 1989. Marine Bivalvia of the Arctic Ocean // Y. Herman (ed.). The Arctic Seas. New York: Van Nostrand. P.303-324.

Filatova Z.A. 1957a. [General review of the fauna of Bivalve molluscs of the Arctic seas] // Trudy Instituta Okeanologii im. P.P. Shirshova. Vol.20. P.3-59 [in Russian].

Filatova Z.A. 1957b. [Zoogeographic division of northern seas on the basis of distribution of bivalves] // Trudy Instituta Okeanologii im. P.P. Shirshova. Vol.23. P.195-215 [in Russian].

Funder S., Bennike O., Böcher J., Israelson C., Petersen K.S., Símonarson L.A. 2001. Late Pliocene Greenland - The Kap København Formation in North Greenland // Bull. Geol. Soc. of Denmark. Vol.48. P.117-134.

Galand P.E., Fevolden S.-E. 2000. Population structure of Chlamys islandica in the Northeast Atlantic - northern stocks compared with a southern relict population // Sarsia. Vol.85. P.183-188.

Galkin Yu.I. 1955. [Gastropod molluscs trochids of Far Eastern and northen seas of the USSR (Family Trochidae)]. Opredeliteli po faune SSSR, izdavaemye zoologicheskim institutom Akademii nauk SSSP. Vol.57. 131 p. [in Russian].

Galkin Yu.I., Voronkov A. Yu. 2001. Class Bivalvia. List of species of free-living invertebrates of Eurasian Arctic seas and adjacent deep waters // Exploration of the fauna of the seas. St.-Petersburg. Vol.51. P.111-114.

Gladenkov Y.B. 1978. [Marine upper Cenozoic of northern regions]. Moscow: Nauka Publ. 194 p. [in Russian].

Gladenkov A.Y., Oleinik A.E., Marincovich L., Jr., Barinov K. 2002. A refined age for earliest opening of Bering Strait // Palaeogeogr. Palaeoclimatol. Palaeoecol. Vol.183. P.321-328.

Gladenkov A.Y, Gladenkov Y.B. 2004. Onset of connections between the Pacific and Arctic Oceans through the Bering Strait in the Neogene // Stratigr. Geol. Correl. Vol.12. P.175-187.

Glibert M., Van de Poel L. 1967. Les Bivalvia fossils du Cénozoique Etranger des collections de l'Institut Roy- 
al des Sciences Naturelles de Belgique. V. Oligondontina. 1 partie: Lucinacea, Cyamiacea, Leptonacea, Dreissenacea, Tellinacea // Mém. Inst. r. Sci. nat. Belg., ser. 2. Vol.83. P.1-152.

Golikov A.N. 1985. [Stages and boundaries of Cenozoic. Analysis of the passage of geological time and development of the fauna of marine gastropods] // Trudy Zool. Inst. AN SSSR. Vol.130. P.31-52 [in Russian].

Gorbunov G.P 1946. [Bottom life of the Novosiberian shoalwaters and the central part of the Arctic Ocean] // Trudy dreifuyustchei ekspeditsii Glavsevmorputi na ledokolnom parokhode "G. Sedov" 1937-1940. Vol.3. P.30-138 [in Russian, English summary].

Grant U.S., Gale H.R. 1931. Catalogue of the marine Pliocene and Pleistocene Mollusca of California and adjacent regions // Mem. San Diego Soc. Nat. Hist. Vol.1. P.1-1036.

Gulliksen B., Palerud R., Brattegard T., Sneli J.-A. 1999. Distribution of marine benthic macro-organisms at Svalbard (including Bear Island) and Jan Mayen. Norwegian Directorate for Nature Management. Research Report for DN 1999-4. Trondheim. 148 p.

Hansen J., Hanken N.-M., Nielsen J.K., Thomsen E. 2011. Late Pleistocene and Holocene distribution of Mytilus edulis in the Barents Sea region and its palaeoclimatic implications // Journal of Biogeography. Vol.38. P.1197-1212.

Hansson H.G. (Comp.). 1998. NEAT (North East Atlantic Taxa): Scandinavian marine Mollusca Check-List // Internet Ed., Aug. 1998. [http://www.tmbl.gu.se].

Hardy S., Carr C., Hardman M., Steinke D., Corstorphine E., Mah C. 2011. Biodiversity and phylogeography of Arctic marine fauna: insights from molecular tools // Marine Biodiversity. Berlin/ Heidelberg: Springer. P.195-210.

Hayasaka S. 1962. Summary of the geology and paleontology of the Atsumi Peninsula, Aichi Prefecture, Japan // Sci. rep. Tohoku Imperial Univ. 2nd series, Geology. P.195-217.

Hayasaka S. 1973. Pliocene marine fauna from Tane-gashima, South Kyushu, Japan // Sci. rep. Tohoku Imperial Univ. 2nd series (Geology). Spec.Vol.6. P.97-108.

Herman J., Marquet R. 2007. Observations paléontologiques réalisées dans les terrains néogènes belges de 1971 à 2004 entre Kallo et Doel, Port d'Anvers, Rive gauche (Flandre-Orientale, Belgique). Le Miocène du Deurganckdok à Doel // Mem. geol. Surv. Belgium. Vol.54. P.1-48.

Honda Y. 1989. Paleogene molluscan faunas from the Kushiro Coal Field, Eastern Hokkaido // Sci. rep. of the Tohoku Imperial Univ. 2nd series (Geology). Vol.60. No.1. P.1-137.

Hopkins D.M., MacNeil F.S. 1960. A marine fauna probably of late probably of late Pliocene age near Kivalina, Alaska // US Geol. Surv. Prof. Pap. Vol.400(B). P.339-342.

Itoigawa J., Ogawa H. 1973. Pleistocene molluscan fauna of the Sakishima Formation, Shima Peninsula, central Japan // Sci. rep. of the Tohoku Imperial Univ. 2nd series (Geology). Spec.Vol.6. P.69-80.

Janssen A.C., Moerdijk P.W., Meijer T. 2003. First record of Megayoldia thraciaeformis (Storer 1838)(Bivalvia) from the Pleistocene of the North Sea Basin // Cainozoic Res. Vol.2. P.139-142.

Janssen A.W. 1972. Die Mollusken-Fauna der Twistringer Schichten (Miocän) von Norddeutschland // Scripta Geol. Vol.10. P.1-96.
Janssen A.W. 1984. Mollusken uit het Mioceen van Winterswijk-miste. Een inventarisatie, met beschrijvingen en afbeeldingen van alle aangetroffen soorten. Koninklijke Nederlandse Natuurhistorische Vereniging, Nederlandse Geologische Vereniging \& Rijksmuseum van Geologie en Mineralogie. Amsterdam. 451 p.

Janssen R., Krylova E.M. 2012. Bivalves of the family Vesicomyidae from the Neogene Mediterranean basin (Bivalvia: Vesicomyidae) // Arch. Mollusk. Bd.141. No.1. P.87-113.

Jimenez A.P., Braga J.C. 1993. Occurrence and taphonomy of bivalves from the Nijar reef (Messinian, Late Miocene, SE Spain) // Palaeogeogr. Palaeoclimatol. Palaeoecol. Vol.102. P.239-251.

Kafanov A.I. 1974. [Composition, systematics and history of development of the Clinocardium group (Mollusca, Cardiidae)] // Zool. Zhurn. Vol.53. No.10. P.14661476 [in Russian].

Kafanov A.I. 1979. [On status of the Arctic marine biogeographic area] // Paleoekologiya soobshestva morskih bespozvonochnyh. Vladivostok: DVNTS Akad. Nauk SSSR. P.100-129 [in Russian].

Kafanov A.I. 1980. Systematics of the subfamily Clinocardiinae Kafanov, 1975 (Bivalvia, Cardiidae) // Malacologia. Vol.19. No.2. P.297-328.

Kafanov A.I. 1987. [Subfamily Mytilinae Rafinesque, 1815 (Bivalvia, Mytilidae) in Cenozoic of the Northern Pacific Region] // Fauna i rasprostranenie molluskov: Severnaya Pacifika i Polyarnyi bassein. Vladivostok: DVNTS Akad. Nauk SSSR. P.65-103 [in Russian].

Kafanov A.I., Ogasawara K. 2003. Neogene and Paleogene Molluscan (Bivalvia) Cenozones of Sakhalin and Kurile Islands // Sci. Rep. Inst. Geosci. Univ. Tsukuba. Vol.24. P.45-79.

Kafanov A.I., Ogasawara K. 2004. Neogene and Paleogene Molluscan (Bivalvia) Cenozones of the Russian Northeast // Sci. Rep. Inst. Geosci. Univ. Tsukuba, Sect B. Vol.25. P.9-45.

Kafanov A.I., Ogasawara K., Marincovich L., Jr. 2001. Checklist and bibliography of the Cenozoic Bivalvia (Mollusca) of Northeastern Asia (Russian Far East), 1968-1999 // Bull. Mizunami Foss. Mus. Vol.28. P.1-138.

Kantor Yu.I., Sysoev A.V. 2005. [Catalogue of molluscs of Russia and adjacent countries]. Moscow: KMK Sci. Press Ltd. 627 p. [in Russian].

Knudsen J. 1985. Abyssal Mollusca of the Arctic Ocean // Journal of Conchology. Vol.32. P.97-107.

Krylov A.V. 2008. [Results of investigation of marine molluscs from the Cenozoic deposits of northeast region of Archangel'sk district] // E.M. Nesterov (ed.). Geologia, geoecologia, evolucionnaya geographia. StPetersburg: Epigraph publ. P.60-62 [in Russian].

Kussakin O.G. 1979. [Marine and brackish-water Isopoda of the cold and temperate waters of the Northern Hemisphere. I. Suborder Flabellifera] // Opredeliteli po faune SSSR [Keys to the fauna of U.S.S.R.]. Vol.122. P.1-472 [in Russian].

Leontjev O.K. 1985. [Morphometry] // A.F. Treshnikov, S.S. Sal'nikov (eds.). Arcticheskii i Jushnui oceany. Leningrad: Nauka Publ. P.17-18 [in Russian].

Lockwood B.L., Sanders J.G., Somero G.N. 2010. Transcriptomic responses to heat stress in invasive and 
native blue mussels (genus Mytilus): molecular correlates of invasive success // J. Exp. Biol. Vol.15. No.213. P.3548-3558.

Lozano Francisco M.C., Vera Pelaez J.L., Guerra-Merchan A. 1993. Arcoida (Mollusca, Bivalvia) del Plioceno de la provincia de Malaga, Espana // Treballs del Museu de Geologia de Barcelona. Vol.3. P.157-188.

Lubinsky I. 1980. Marine bivalvia molluscs of the Canadian Central and Eastern Arctic: faunal composition and zoogeography // Can. Bull. Fish. Aquat. Sci. Vol.207. P.1-111.

Lumley H., de. 1988. La stratigraphie du remplissage de la Grotte du Vallonnet // L'Anthropologie. T.92. No.2. P.407-428.

MacNeil F.S. 1957. Cenozoic Megafossils of Northern Alaska// US Geol. Surv. Prof. Pap. Vol.294(C). P.99126.

MacNeil F.S. 1965. Evolution and distribution of the genus Mya, and Tertiary migrations of Mollusca // US Geol. Surv. Prof. Pap. Vol.483(G). 51 p.

Makowska A. 1986. [Pleistocene seas in Poland; sediments, age and paleogeography] // Prace Instytutu Geologicznego. Vol.120. P.74 [in Polish, with English summary].

Mankiewicz C. 1996. The Middle to Upper Miocene carbonate complex of Nijar, Almeria Province, southeastern Spain // SEPM Concepts in Sedimentology and Paleontology. Vol.5. P.141-157.

Marasti R. 1973. La fauna tortoniana del T. Stirone (limite Parmense-Piacentino) // Boll. Soc. Paleo. Italiana. Vol.12. No.1. P.76-120.

Marincovich L. 1983. Molluscan paleontology, paleoecology, and North Pacific correlations of the Miocene Tachilni Formation, Alaska Peninsula, Alaska // Bull. American Paleo. Vol.84. No.317. P.1-155.

Marincovich L., Jr., Gladenkov A.Y. 1999. Evidence for an early opening of the Bering Strait// Nature. Vol.397. P.149-151.

Marquet R. 1995. A revision of the Caenozoic Pinnidae from Belgium (Mollusca, Bivalvia) // Bull. Inst. R. Sci. Nat. Belg. Sci. Terre. Vol.65. P.241-256.

Marquet R. 2002. The Neogene Amphineura and Bivalvia (Protobranchia and Pteriomorphia) from Kallo and Doel(Oost Vlaanderen, Belgium)//Palaeontos. Vol.2. P.1-100.

Marquet R. 2005. The Neogene Bivalvia (Heterodonta and Anomalodesmata) and Scaphopoda from Kallo and Doel (Oost Vlaanderen, Belgium)// Palaeontos. Vol.6. P.1-142.

Meijer T. 1993. Stratigraphical notes on Macoma (Bivalvia) in the southern part of the North Sea Basin and some remarks on the arrival of Pacific species // Scripta Geol., Spec. Issue 2. P.297-312.

Merklin R.L., Petrov O.M., Amitrov O.V. 1962. [AtlasGuide to the molluscs of the Quaternary deposits of the Chukotsk Peninsula]. Moscow: Izdatel'stvo Akademii Nauk SSSR. 56 p. [in Russian].

Merklin R.L., Zarkhidze D., Il'ina L.B. 1979. [Key to identification of marine Pliocene-Pleistocene molluscs of the North-Eastern region of the European part of the USSR] // Trudy PIN AN SSSR. Moscow: Nauka Publ. Vol.173. 96 p. [in Russian].

Miller R.D., Dobrovolny E. 1959. Surficial geology of Anchorage and vicinity, Alaska // US Geol. Surv. Bull. No.1093. 128 p.
Miller W. 1990. Checklist of megafossils from the James City Formation (Lower Pleistocene) at Johnson Point, Craven County, North Carolina // Tulane Studies in Geology and Paleontology. Vol.22. P.93-100.

Mironov A.N. 2006. [Centers of marine fauna redestribution] // Zool. Zhurn. Vol.85. No.1. P.3-17 [in Russian, English summary].

Mironov A.N., Dilman A.B. 2010. [Effect of the East Siberian barrier on the echinoderm dispersal in the Arctic Ocean] // Okeanologia. Vol.50. No.3. P.342355 [in Russian, English summary] [English translation: Oceanology. Vol.50. No.3. P.342-355].

Moths H., Albrecht F., Stein G. 2010. Die molluskenfauna (Hermmorium, Untermiozän) aus der Kiesgrube kirnke bei Werder (Nordwest-Niedersachsen) // Palaeofocus. Vol.3. P.1-155.

Moore E.J. 1963. Miocene marine molusks from the Astoria formation in Oregon // US Geol. Surv. Prof. Pap. Vol.419. P.1-109.

Nakashima R. 2005. An overview of the Late Cenozoic bivalve Panomya in Japan // J. Paleont. Vol.79. No.3. P.500-519.

Naumov A.D. 2006. [Clams of the White Sea. Ecological and faunistic analysis]. St-Petersburg. 367 p. [in Russian]

Nesis K.N. 1963. The routes, and the periods of formation, of the interrupted area of distribution of amphiboreal species of marine bottom animals // Deep-Sea Research. Vol.10. No.4. P.498-506.

Nesis K.N. 1965. Ecology of Cyrtodaria siliqua and history of the genus Cyrtodaria (Bivalvia: Hiatellidae) // Malacologia. Vol.3. P.197-210.

Nikula R., Strelkov P., Väinölä R. 2007. Diversity and trans-arctic invasion history of mitochondrial lineages in the Nrth Atlantic Macoma balthica complex (Bivalvia: Tellinidae) // Evolution. Vol.61. No.4. P.928-941.

Norton P.E. 1967. Marine Molluscan Assemblages in the Early Pleistocene of Sidestrand, Bramerton and the Royal Society Borehole at Ludham, Norfolk // Phil. Trans. Roy. Soc. London. Ser. B, Biol. Sci. Vol.253. No.784. P.161-200.

O'Hara S., Nemoto N. 1988. Pectinids from the Taga Group of the Joban Coalfield // Saito Ho-on Kai Special Publication (Professor T. Kotaka Commemorative Volume). P.481-496.

Okamoto K., Ibaraki M. 1988. Early Pleistocene fossil Mollusca from the Sea of Japan off Yamaguchi Prefecture // Saito Ho-on Kai Special Publication (Professor T. Kotaka Commemorative Volume). P.523-532.

Petersen H.G. 2001. Studies on some Arctic and Baltic Astarte species (Bivalvia, Mollusca) // Bioscience. Vol.52. P.1-71.

Philippi R.A. 1844. Enumeratio molluscorum Siciliae cum viventium tum in tellure tertiaria fossilium, quae in itinere suo observavit. Halle: Eduard Anton.Vol.2. 303 p.

Popov S.V., Gontsharova I.A., Nikolov P.I., Studencka B. 1996. Bivalve Molluscs from the Badenian of Northwestern Bulgaria // Paleo. Journal. Vol.30. No.2. P.157-166.

Ragaini L., Cantalamessa G., Di Celma C., Didaskalou P., Impiccini R., Lori P., Marino M., Potetti M., Ragazzi- 
ni S. 2007. First Emilian record of the boreal-affinity bivalve Portlandia impressa Perri, 1975 from Montefiore dell'Aso (Marche, Italy) // Boll. Soc. Italiana. Vol.45. No.2-3. P.227-234.

Reed F.R.C. 1935. Notes on the Neogene faunas of Cyprus, II // Ann. Mag. Nat. Hist. Vol.15. No.85. P.1-31.

Remia A., Taviani M. 2005. Shallow-buried Pleistocene Madrepora-dominated coral mounds on a muddy continental slope, Tuscan Archipelago, NE Tyrrhenian Sea // Facies. Vol.50. No.3-4. P.419-425.

Richards H.G. 1962. Studies on the marine Pleistocene: Part II. The marine Pleistocene mollusks of eastern North America // Trans. Americ. Phil. Soc. Vol.52. No.3. P.1-142.

Richling I. 2000. Arktische Bivalvia - eine taxonomische Bearbeitung auf Grundlage des Materials der Expeditionen Transdrift 1 und ARK IX/4 (1993) in das Laptevmeer // Schriften zur Malakozoologie. Vol.15. P.1-93.

Scarlato O.A. 1981. [Bivalve molluses of the temperate latitudes of the western part of the Paciûc Ocean]. Leningrad: Nauka Publ. 479 p. [in Russian].

Scarlato O.A., Kafanov A.I. 1988. [Additions to the bivalve mollusks fauna of Far Eastern seas of the USSR] // Zool. Zhurn. Vol.67. No.6. P.937-942 [in Russian].

Schiøtte T. 2005. Marine, benthic molluscs common to North Greenland and the Faroes: A first investigation of a previously unrecognized, North Atlantic fauna element // BIOFAR Proceedings. P.94-108.

Schneider S., Witt W., Yigitbas E. 2005. Ostracods and bivalves from an Upper Pleistocene (Tyrrhenian) marine terrace near Altinova (Izmit Province, Turkey) // Zitteliana. Vol.45. P.87-114.

Sher A. 1999. Traffic lights at the Beringian crossroads // Nature. Vol.397. P.103-104.

Simonarson L.A., Petersen K.S., Funder S. 1998. Molluscan palaentology of the Pliocene-Pleistocene Kap Kobenhavn Formation, North Greenland// Meddelelser om Grønland, Geoscience. Vol.36. 104 p.

Sirenko B.I. 2001. List of species of free-living invertebrates of Eurasian Arctic Seas and adjacent deep waters // Explor. Fauna Seas. Vol.51. P.1-76.

Sirenko B.I., Vassilenko S.V., Petryashov V.V. 2009. Distribution patterns for shelf species of Eurasian Arctic Seas // S.V. Vassilenko, V.V. Petryashov (eds.). Illustrated keys to free-living Invertebrates of Eurasian Arctic Seas and adjacent deep waters. University of Alaska Fairbanks: Alaska Sea Grant. Vol.1. P.179-186.

Smirnov A.V. 1994. Arctic echinoderms: composition, distribution and history of the fauna // Echinoderms through time. Rotterdam: A.A. Balkema. P.135-142.

Sneli J.-A., Schiøtte T., Jensen K.R., Wikander P.B., Stokland Ø., Sørensen J. 2005. The marine Mollusca of the Faroes // Fróðskaparrit Supplementum. Vol.42. P.15-176.

Soot-Ryen T. 1932. Pelecypoda, with a discussion of possible migrations of Arctic pelecypods in Tertiary times. Norwegian North Polar Expedition. Scientific Results. Bergen: Geofysisk Institut. Vol.5. No.12. 35 p.

Studencka B. 1994. Middle Miocene bivalve faunas from the carbonate deposits of Poland (Central Paratethys) // Geologie Mediterraneenne. Vol.11. No.12. P.137-145.
Studencka B., Gontsharova I.A., Popov S.V. 1998. The bivalve faunas as a basis for reconstruction of the Middle Miocene history of the Paratethys // Acta Geol. Pol. Vol.48. No.3. P.285-342.

Svitoch A.A. 2003. [Marine pleistocene of Russian coast]. Moscow: GEOS. 361 p. [in Russian].

Takayasu K. 1986. Characteristics of the Miocene molluscan assemblages from central San-in District, Southwest Honshu, Japan // Monog. Mizunami Foss. Mus. Vol.6. P.155-169.

Väinölä R. 2003. Repeated trans-Arctic invasions in littoral bivalves: molecular zoogeography of the Macoma balthica complex // Mar. Biol. Vol.143. P.935-946.

Väinölä R., Strelkov P. 2011. Mytilus trossulus in Northern Europe // Mar. Biol. Vol.158. P.817-833.

Vandenberghe N., Laga P., Louwye Vanhoorne S., Marquet R., De Meuter F., Wouters K., Hagemann H. W. 2005. Stratigraphic interpretation of the Neogene marine-continental record in the Maaseik well (49W0220) in the Roer Valley Graben, NE Belgium // Mem. Geol. Surv. Belgium. Vol.52. P.1-39.

Varvio S.L., Koehn R.K., Väinölä R. 1988. Evolutionary genetics of the Mytilus edulis complex in the North Atlantic Region // Mar. Biol. Vol.98. P.51-60.

Vera-Peláez J.L., Lozano-Francisco M.C., Ramos Fernández J., Cortéz Sánchez Y.M. 2004. Molluscos del Tirreniense (Pleistoceno Superior) de la Playa la ArañaCala de Moral (Málaga) // Revista Española de Paleontología. Vol.19. No.2. P.251-259.

Vermeij G.J. 1991. Anatomy of an invasion: the transArctic interchange // Paleobiology. Vol.17. P.281-307.

Wagner F.J.E. 1970. Faunas of the Pleistocene Champlain Sea // Bull. Geol. Surv. Canada. Vol.181. P.1-104.

Ward L.W. 1993. The G.D Harris 1890 manuscript on the stratigraphy of Miocene and Pliocene beds at Yorktown, Virginia // Paleo. Res. Inst. Spec. Publ. Vol.20. P.1-118.

Ward L.W., Blackwelder B.W. 1987. Late Pliocene and Early Pleistocene Mollusca from the James City and Chowan River Formations at Lee Creek Mine // Smithsonian Contributions to Paleobiology. Vol.61. P.113283.

Warén A. 1989. Taxonomic comments on some protobranch bivalves from the northeastern Atlantic // Sarsia. Vol.74. No.4. P.223-297.

Warén A. 1991. New and little known Mollusca from Iceland and Scandinavia // Sarsia. Vol.76. No.1-2. P.23-124.

Wienrich G. 1999. Die Fauna des marinen Miozäns von Kevelaer (Niederrhein). Band 2 Bivalvia, Scaphopoda, Cephalopoda, Bryozoa, Annelida, Brachiopoda. Leiden: Backhuys Publishers. H.2. S.188-384.

Woods S.V. 1850. A monograph of the Crag Mollusca, or, descriptions of shells from the middle and upper tertiaries of the East of England. Part II. Bivalves. 302 p.

Wood S.V. 1879. Monograph of the Crag Mollusca with descriptions of shells from the upper tertiaries of the east of England. 58 p.

Zibrowius H. 1995. The "southern" Astroides calycularis in the Pleistocene of the northern Mediterranean - an indicator of climatic changes (Cnidaria, Scleractinia) // Geobios. Vol.28. No.1. P.9-16. 